\title{
Critical force in active microrheology
}

\author{
M. Gruber ${ }^{1},{ }^{1}$ A. M. Puertas, ${ }^{2}$ and M. Fuchs ${ }^{1}{ }^{1, *}$ \\ ${ }^{1}$ Fachbereich Physik, Universität Konstanz, 78457 Konstanz, Germany \\ ${ }^{2}$ Departamento de Física Aplicada, Universidad de Almería, 04.120 Almería, Spain
}

(Received 23 October 2019; published 30 January 2020)

\begin{abstract}
Soft solids like colloidal glasses exhibit a yield stress, above which the system starts to flow. The microscopic analogon in microrheology is the untrapping or depinning of a tracer particle subject to an external force exceeding a threshold value in a glassy host. We characterize this delocalization transition based on a bifurcation analysis of the corresponding mode-coupling theory equations. A schematic model that allows analytical progress is presented first, and the full physical model is studied numerically next. This analysis yields a continuous dynamic transition with a critical power-law decay of the probe correlation functions with exponent $-1 / 2$. To compare with simulations with a limited duration, a finite-time analysis is performed, which yields reasonable results for not-too-small wave vectors. The theoretically predicted findings are verified by Langevin dynamics simulations. For small wave vectors we find anomalous behavior for the probe position correlation function, which can be traced back to a wave-vector divergence of the critical amplitude. In addition, we propose and test three methods to extract the critical force from experimental data, which provide the same value of the critical force when applied to the finite-time theory or simulations.
\end{abstract}

DOI: 10.1103/PhysRevE.101.012612

\section{INTRODUCTION}

The yield stress in ductile solids is the crossover between linear elasticity and plastic deformations. However, in soft matter systems, the yield stress is more conveniently defined as the minimum stress needed to provoke the flow of the system [1]; in a fluid this minimum stress is zero, while it is generically finite for a soft matter solid. Starting from simple constitutive relations, different models have been developed describing the yield stress in terms of the microscopic characteristics of the system [1]; in particular, let us mention the soft glassy rheology model [2], the shear transformation zone model [3], or the mode coupling approach [4]. Our approach to the problem is based on the application of a localized stress, induced by a colloidal tracer that is pulled externally, namely, active microrheology.

Microrheology was proposed more than 20 years ago as a technique to access the rheological properties at the microscopic scale, monitoring the dynamics of colloidal tracers introduced in the sample [5]. However, it was soon acknowledged that the technique could be significantly improved if the tracer is pulled externally (active microrheology), as both the linear and nonlinear regimes can be studied [6,7]. Several models for active microrheology have been presented, based on an effective medium approach [8], the two-particle Smoluchowski equation (for low densities) for stationary [9] and transient regimes [10], the mode-coupling approach (applicable to high densities) [11], the continuous time random walk model $[12,13]$, a kinetically constrained model $[13,14]$. Simulation studies [15-17] and first experimental studies confirmed that the dynamics becomes highly anomalous in glass-forming dispersions $[18,19]$. For

\footnotetext{
*matthias.fuchs@uni-konstanz.de
}

strongly interacting systems there is a depinning [20] of elastic manifolds or a untrapping/delocalization $[21,22]$ of the probe particle. In the following, we will call this a delocalization transition, following the terminology of previous publications $[11,22,23]$.

In a previous study, active microrheology in a colloidal glass was analyzed with a model based on the mode-coupling approximation, and tested against simulations [22]. It was found in that work that there are two regimes for the dynamics of the tracer: a localized regime, found when the external force is small, and where the tracer is trapped and localized in the cage formed by its own neighbours, and a delocalized regime, when the force is large enough to break the cage, and the tracer exhibits motion over long distances. The properties of the localized regime were studied in detail in that work, and confirmed by the simulations. The focus of the present study, is the examination of the system's behavior at the crossover between these two regimes.

We present in this paper the properties of the critical force separating both regimes within the model, and use them to nail down the critical force in the simulations and confirm the predictions of the model. In particular, it is predicted that the long-time limit of the tracer position correlation function (nonergodicity parameter) decays linearly with the external force, being zero at the critical force (identified as a type $A$ transition within mode-coupling theory). At the critical force, the correlation function decays as function of time according to a power-law with exponent $-1 / 2$, and a prefactor that coincides with the slope of the nonergodicity parameter with the external force. For small wave vectors perpendicular to the external force some anomalies are found in the theory. Because the long time limit is generally unreachable in the simulations, we test within the theory, how the predictions are altered if values available at finite times are considered. Our simulation results confirm the predictions of the model, 
validating the analysis and providing a toolbox to estimate the critical force. This can prove useful to identify the critical force in experimental systems [19].

\section{THEORY}

In active microrheology we consider a spherical probe particle with diameter $d$, which is pulled by a constant external force $\boldsymbol{F}_{\text {ext }}=F_{\text {ext }} \hat{\boldsymbol{e}}_{z}$ through a colloidal suspension of spheres of the same diameter, the host. All particles are subject to Brownian motion induced by the suspending fluid, but hydrodynamic interactions are neglected. Our description is based on the displacement distribution function $G^{s}(\boldsymbol{r}, t)$ (also called the self-part of the van-Hove-function) and, more precisely, on its spatial Fourier transform

$$
\Phi_{\boldsymbol{q}}^{s}(t)=\int e^{i \boldsymbol{q} \cdot \boldsymbol{r}} G^{s}(\boldsymbol{r}, t) d \boldsymbol{r}
$$

also called probe correlator.

\section{A. Model}

The dynamics of this probe correlation function is derived from a microscopic overdamped Smoluchowski equation within the framework of mode-coupling theory (MCT) [22]. It is given by an integrodifferential equation

$$
0=\partial_{t} \Phi_{q}^{s}(t)+\Gamma_{\boldsymbol{q}} \Phi_{q}^{s}(t)+\int_{0}^{t} m_{\boldsymbol{q}}\left(t-t^{\prime}\right) \partial_{t^{\prime}} \Phi_{q}^{s}\left(t^{\prime}\right) d t^{\prime},
$$

with initial condition $\Phi_{q}^{s}(0)=1 . \quad \Gamma_{\boldsymbol{q}}=q^{2}-i \boldsymbol{q} \cdot \boldsymbol{F}_{\mathrm{ext}} \mathrm{de}-$ scribes the free decay of the correlator as if no other particles were present and the memory kernel $m_{q}$ accounts for the interactions between the probe and the bath. $m_{q}$ is given by a functional of the probe and the host correlators using MCT. For the scaling analysis it is convenient to introduce the Laplace transformed quantities given by

$$
\tilde{A}(s)=\int_{0}^{\infty} d t e^{-s t} A(t)
$$

The equation of motion Eq. (2) then reads

$$
\tilde{\Phi}_{\boldsymbol{q}}^{s}(s)=\left(s+\frac{\Gamma_{\boldsymbol{q}}}{1+\tilde{m}_{\boldsymbol{q}}(s)}\right)^{-1} \text {. }
$$

Using the cylindrical symmetry of the system around the force direction, we find (choosing $\boldsymbol{q}=\left(q_{x}, 0, q_{z}\right)$ without loss of generality)

$$
\frac{\Gamma_{\boldsymbol{q}}}{1+\tilde{m}_{\boldsymbol{q}}}=\frac{\Gamma_{\boldsymbol{q}}^{x}\left(1+\tilde{m}_{\boldsymbol{q}}^{z z}\right)-\Gamma_{\boldsymbol{q}}^{x z} \tilde{m}_{\boldsymbol{q}}^{x z}+\Gamma_{\boldsymbol{q}}^{z}\left(1+\tilde{m}_{\boldsymbol{q}}^{x x}\right)}{\left(1+\tilde{m}_{\boldsymbol{q}}^{x x}\right)\left(1+\tilde{m}_{\boldsymbol{q}}^{z z}\right)-\tilde{m}_{\boldsymbol{q}}^{x z} \tilde{m}_{\boldsymbol{q}}^{x z}},
$$

with $\Gamma_{\boldsymbol{q}}^{x}=q_{x}^{2}, \Gamma_{\boldsymbol{q}}^{z}=q_{z}^{2}-i q_{z} F_{\mathrm{ext}}, \Gamma_{\boldsymbol{q}}^{x z}=2 q_{x} q_{z}-i q_{x} F_{\mathrm{ext}}$, and the primitive memory functionals

$$
m_{\boldsymbol{q}}^{\alpha \beta}\left[\boldsymbol{\Phi}^{s}(t), \boldsymbol{\Phi}(t)\right]=\frac{1}{(2 \pi)^{3}} \int d \boldsymbol{k} p_{\alpha} p_{\beta} \frac{\left(S_{p}^{s}\right)^{2}}{n S_{p}} \Phi_{\boldsymbol{k}}^{s}(t) \Phi_{p}(t),
$$

with $\boldsymbol{p}=\boldsymbol{q}-\boldsymbol{k} . S_{p}=\left\langle\rho_{\boldsymbol{p}}, \rho_{\boldsymbol{p}}\right\rangle / N$ is the static bath structure factor, $S_{p}^{s}=\left\langle\rho_{p}^{s}, \rho_{p}\right\rangle$ the static probe-bath structure factor with the Fourier transformed bath particle density $\rho_{\boldsymbol{p}}$ and the probe density $\rho_{p}^{s}$ (see p. 133 and p. 322 in Ref. [24]). $\Phi_{p}(t)$ is the bath correlation function. With the structure factor obtained in the Percus-Yevick approximation for hard spheres, the host presents a glass transition within $\mathrm{MCT}$ at $\varphi_{g}=0.516$. For this work, we chose a packing fraction of $\varphi=0.537$, which is about $4 \%$ above the glass transition.

Upon increasing the force, this model exhibits a delocalization transition, where the long time limit $f_{q}^{s}:=\lim _{t \rightarrow \infty} \Phi_{q}^{s}(t)$ changes from $f_{\boldsymbol{q}}^{s}>0$ (nonergodic, localized behavior) for small forces to $f_{\boldsymbol{q}}^{s}=0$ (ergodic, delocalized behavior) for forces above the critical force $F_{c}$. With this criterion we can determine the critical force unambiguously from the MCT calculations [22,25]. However, this definition requires the evaluation of correlation functions at infinite times, which are not accessible neither in experiments nor in simulations. The aim of this work is to characterize this transition to provide and test suitable methods for experimental systems and simulations and to show that they consistently yield the same critical force.

\section{B. Numerical details}

The numerical solution algorithm is described in Appendix $\mathrm{C}$ of Ref. [22] and in more detail in Chap. 3 of Ref. [25]. We choose a cutoff of $q_{\max } d=14$ for $q_{r}$ and $q_{z}$ on a uniform grid from 0 to $q_{\max } d$ with step size $\Delta q d=0.5$ with $N=29$ points, which is refined toward 0 by adding $N_{\log }=10$ nonuniformly spaced grid points given by $2^{-i} \Delta q d(i=1, \ldots, 10)$. This choice allows sufficient resolution for long-ranged structures as well as the microscopic structures, while allowing reasonable computing times $(39 \times 39$ correlation functions have to be calculated) and preventing numerical issues [26]. The dynamical solutions are obtained using the decimation algorithm with an initial step size of $\Delta t d^{2} / D_{0}=10^{-8}$ on a grid with 1024 grid points using up to 45 decimation steps. The bath correlator is calculated on the same time grid and the usual grid in $q$-space with cutoff $q d=65$ and 512 grid points. The critical force depends slightly on the discretization. For the parameters above (and a packing fraction of $\varphi=0.537)$ the critical force is given by $F_{c}=(44.79 \pm$ 0.01 ) $k_{\mathrm{B}} T / d$. For the analysis of the critical dynamics, we use $N_{\log }=25$ points in the nonuniform part of the grid for a better resolution of the critical force, which is given by $F_{c}=(44.7815 \pm 0.001) k_{\mathrm{B}} T / d$. We tested two algorithms for the time integration based on (i) the integral equation and (ii) the integrodifferential equation representation for the effective memory function. They give the same results, but differ in the regions of stability; see Chap. 3 of Ref. [25] for details.

\section{BIFURCATION ANALYSIS}

In this section, we investigate the behavior of the probe correlation function $\Phi_{q}^{s}(t)$ close to the delocalization transition, viz. a kind of depinning transition. This transition is characterized by the long-time limits $f_{q}^{s}:=\lim _{t \rightarrow \infty} \Phi_{q}^{s}(t)$. In the glass, i.e., for $\varphi>\varphi_{g}$, we find $f_{q}^{s} \neq 0$ for small external forces, while $f_{q}^{s}=0$ if the force is large enough. The smallest force for which $f_{q}^{s}=0$ is the critical force and determines the delocalization transition [22]. The predictions for the behavior of the correlation function close to this critical point will 
provide us with several means to characterize the critical force in simulations and experiments.

Starting point for the bifurcation analysis is the Laplace space version of the equations of motion Eq. (4). To make analytic progress, we approximate the bath correlation function by its long time limit $\Phi_{q}(t) \approx f_{q}:=\lim _{t \rightarrow \infty} \Phi_{q}(t)$. This is a good approximation if the evolution of the probe correlation function decays slower than the bath. Furthermore, this procedure leaves the long-time limit unchanged so that the long time limit will be correct in any case. With this approximation, the primitive memory functionals become linear in $\boldsymbol{\Phi}^{s}(t)$ so that we can directly insert the Laplace transformed taggedparticle correlator $\tilde{\Phi}_{q}^{s}(s)$. To facilitate a discussion of the full equations, we will first perform the bifurcation analysis of a simplified version, a schematic model.

\section{A. Schematic model}

A schematic model corresponding to the full MCT equations was designed by Gustavo Abade modifying previous schematic models [21,27]. It focuses on the characteristic time-dependent behavior of the (complex-valued) parallel and (real-valued) perpendicular modes of the probe-bath correlation function. In the following they are summarized in the vector $\phi(t)=\left(\phi_{\|}(t), \phi_{\perp}(t)\right)$ with $\phi_{\|}(t) \in \mathbb{C}$ and $\phi_{\perp}(t) \in \mathbb{R}$. The equations of motion are given by

$$
\tau_{i} \partial_{t} \phi_{i}+\phi_{i}(t)+\int_{0}^{t} m_{i}\left(t-t^{\prime}\right) \partial_{t^{\prime}} \phi_{i}\left(t^{\prime}\right) d t^{\prime}=0,
$$

for $i \in\{\|, \perp\}$. They have the same structure as the full model in Eq. (2) (multiplied by the timescale $\tau_{i}=\Gamma_{i}^{-1}$ ). This includes the complex valued relaxation times for the taggedparticle correlator $\tau_{\|}=\tau^{s}\left(1-i \kappa_{\|} F_{\text {ext }}\right)^{-1}$ and $\tau_{\perp}=\tau^{s}$, where $\tau^{s}$ describes the relaxation time without external force.

To mimic the couplings introduced by the memory functionals, we have to find an appropriate simplification of Eq. (5). For the parallel direction (i.e., $q_{x}=0$ ) it reduces to the condition $m_{q}=m_{q}^{z z}$ so that we can define for the parallel direction $m_{\|}(t)=\mathcal{F}_{\|}[\boldsymbol{\phi}(t)]$ as in Ref. [27] via

$$
\mathcal{F}_{\|}(\boldsymbol{x})=\frac{\left(v_{1}^{s} x_{\|}^{*}+v_{2}^{s} x_{\perp}\right) f_{b}}{1-i \kappa_{\|} F_{\text {ext }}},
$$

where $v_{1}^{s}$ and $v_{2}^{s}$ describe the coupling of the perpendicular and the parallel component to the bath mode, while $\kappa_{\perp}$ (appearing in the next equation) and $\kappa_{\|}$describe the coupling of the probe correlators to the force. $f_{b}$ is the nonergodicity parameter of the $F_{12}$ model, a one-component MCT-model with a second order memory kernel polynomial (cf. Eq. (4.32b) on p. $202 f$ in Ref. [24]) and describes the frozen-in glass structure of the bath. The star* denotes complex conjugation. Hereby, we just replaced the memory functional by a simple polynomial, which is the standard procedure for schematic MCT models (see, e.g., p. 202ff in Ref. [24]). Furthermore, this memory functional coincides with the parallel memory functional of the schematic model discussed by Gnann et al. in Ref. [27], which is itself an extension of the one-component schematic model of Gazuz [21].

For the perpendicular direction (i.e., $q_{z}=0$ ), the equation of motion Eq. (5) for the effective memory function $m_{\perp}(t)$ does not reduce to a simple memory function as was the case in the model in Ref. [27]. Instead, it is nonlocal in time given by the integrodifferential equation

$$
\begin{aligned}
\tau_{\perp} m_{\perp}(t)+\int_{0}^{t} m_{\perp}\left(t-t^{\prime}\right)\left[m_{\perp}^{z z}\left(t^{\prime}\right)+i \kappa_{\perp} F_{\mathrm{ext}} m_{\perp}^{x z}\left(t^{\prime}\right)\right] d t^{\prime} \\
=\tau_{\perp}\left[m_{\perp}^{x x}(t)-i \kappa_{\perp} F_{\mathrm{ext}} m_{\perp}^{x z}(t)\right]+\int_{0}^{t} m_{\perp}^{x x}\left(t-t^{\prime}\right) m_{\perp}^{z z}\left(t^{\prime}\right) d t^{\prime} \\
\quad-\int_{0}^{t} m_{\perp}^{x z}\left(t-t^{\prime}\right) m_{\perp}^{x z}\left(t^{\prime}\right) d t^{\prime}
\end{aligned}
$$

with the local memory functionals $m_{\perp}^{\alpha \beta}(t)=\mathcal{F}_{\perp}^{\alpha \beta}[\phi(t)]$ given by

$$
\begin{aligned}
& \mathcal{F}_{\perp}^{x x}(\boldsymbol{x})=\left(v_{1}^{s} x_{\perp}+v_{2}^{s} \operatorname{Re}\left\{x_{\|}\right\}\right) f_{b}, \\
& \mathcal{F}_{\perp}^{x z}(\boldsymbol{x})=-i v_{2}^{s} \operatorname{Im}\left\{x_{\|}\right\} f_{b}, \\
& \mathcal{F}_{\perp}^{z z}(\boldsymbol{x})=v_{2}^{s} \operatorname{Re}\left\{x_{\|}\right\} f_{b} .
\end{aligned}
$$

Since the perpendicular correlation function is real valued, this requires the effective perpendicular memory function to be real as well. The symmetries of the full primitive memory functionals suggest the given couplings to the real or imaginary part only.

The schematic model defined by Eqs. (7)-(10) has the free parameters $\tau^{s}, \kappa_{\|}, \kappa_{\perp}, F_{\text {ext }}, v_{1}^{s}, v_{2}^{s}$, and $f_{b}$. The bath nonergodicity parameter $f_{b}$ will be parametrized through the distance $\varepsilon$ to the critical point (viz. glass transition) [28]. The parameter $F_{\text {ext }}$ will be used as analogon to the external force in the full model to drive the system through the delocalization transition. Time will be expressed in units of $\tau^{s}$, which is equivalent to setting $\tau^{s}=1$.

\section{B. Beta-scaling analysis of the schematic model}

For the beta-scaling analysis, we rewrite the equations of motion in terms of the real valued quantities $\phi_{1}:=\operatorname{Re} \phi_{\|}$, $\phi_{2}:=\operatorname{Im} \phi_{\|}$and $\phi_{3}:=\phi_{\perp}$ and obtain in Laplace space

$$
\begin{aligned}
-\frac{\phi_{1}(s)\left(\phi_{1}(s) s-1\right)+\phi_{2}^{2}(s) s}{\phi_{2}^{2}(s) s^{2}+\left(\phi_{1}(s) s-1\right)^{2}} & =\sum_{i=1}^{3}\left(V_{\|}^{\mathrm{re}}\right)_{i} \phi_{i}(s)+\tau_{1}, \quad(11 \mathrm{a}) \\
\frac{\phi_{2}(s)}{\phi_{2}^{2}(s) s^{2}+\left(\phi_{1}(s) s-1\right)^{2}} & =\sum_{i=1}^{3}\left(V_{\|}^{\mathrm{im}}\right)_{i} \phi_{i}(s)+\tau_{2}, \quad(11 \mathrm{~b}) \\
\frac{\phi_{3}(s)}{1-s \phi_{3}(s)}= & \frac{\sum_{i, j=1}^{3}\left(W_{\perp}\right)_{i j} \phi_{i}(s) \phi_{j}(s)}{\tau_{3}+\sum_{i=1}^{3}\left(U_{\perp}\right)_{i} \phi_{i}(s)} \\
& +\frac{\sum_{i=1}^{3}\left(V_{\perp}\right)_{i} \phi_{i}(s)}{\tau_{3}+\sum_{i=1}^{3}\left(U_{\perp}\right)_{i} \phi_{i}(s)}+\tau_{3} .
\end{aligned}
$$

Note that the tildes to indicate Laplace space values have been dropped for the ease of notation. The scalars $\tau_{i}$, the vectors $V_{\|}^{\text {re }}, V_{\|}^{\text {im }}, V_{\perp}, U_{\perp}$ and the symmetric matrix $W_{\perp}$ contain combinations of the model parameters as introduced in Chap. 6 of Ref. [25] and summarized in Appendix A.

Using the final value theorem $\lim _{t \rightarrow \infty} \phi_{i}(t)=$ $\lim _{s \rightarrow 0} s \tilde{\phi}_{i}(s)$ we can identify the determining equation for the nonergodicity parameters $f_{i}:=\lim _{t \rightarrow \infty} \phi_{i}(t)$ by multiplication of Eq. (11) by $s$ and taking the limit $s \rightarrow 0$. The nonergodicity parameters are then given by the roots of 


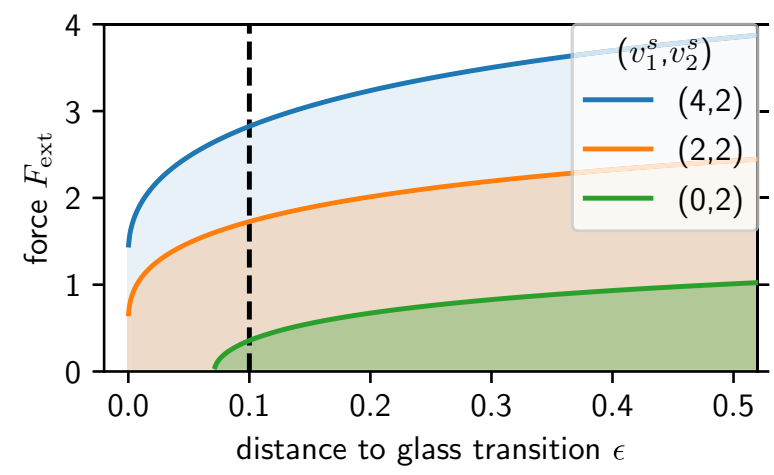

FIG. 1. Phase diagram of the schematic model with $\kappa_{\|}=\kappa_{\perp}=1$ for different couplings. The solid lines show the critical forces for the given parameters. In the shaded regions below the solid lines, the solutions are nonergodic, while they are ergodic above. Different values of $v_{1}^{s}$ for $v_{2}^{s}=2$ are presented, as labeled. According to Eq. (13) there are only quantitative changes on varying $v_{2}^{s}$, but the shape is not affected. The dashed line shows the parameter space for the analysis in Fig. 2.

the following set of nonlinear equations

$$
\begin{aligned}
& J_{1}(\boldsymbol{x})=-\frac{x_{1}\left(x_{1}-1\right)+x_{2}^{2}}{x_{2}^{2}+\left(x_{1}-1\right)^{2}}-\sum_{i=1}^{3}\left(V_{\|}^{\mathrm{re}}\right)_{i} x_{i}, \\
& J_{2}(\boldsymbol{x})=\frac{x_{2}}{x_{2}^{2}+\left(x_{1}-1\right)^{2}}-\sum_{i=1}^{3}\left(V_{\|}^{\mathrm{im}}\right)_{i} x_{i}, \\
& J_{3}(\boldsymbol{x})=\frac{x_{3}}{1-x_{3}}-\frac{\sum_{i, j=1}^{3}\left(W_{\perp}\right)_{i j} x_{i} x_{j}}{\sum_{i=1}^{3}\left(U_{\perp}\right)_{i} x_{i}} .
\end{aligned}
$$

In the glass $(\varepsilon>0)$ for vanishing force $F_{\text {ext }}$ and for not too small couplings $v_{1}^{s}, v_{2}^{s}$, there is a nontrivial solution $f_{i} \neq 0$ of this set of Eqs. (12). Increasing the force, there is a critical force $F_{c}$, above which there exists only the trivial solution $f_{i}=0$. This is visualized for some parameters in Fig. 1. If the coupling to the bath is too weak (e.g., $v_{1}^{s}=0, v_{2}^{s}=2$ ), the critical force appears only deep in the glass. The lines for the critical forces for $\kappa_{\|}=\kappa_{\perp}=\kappa$ were obtained analytically in Sec. 6.2.4 in Ref. [25], which is reproduced in Appendix B and read

$$
F_{c}=\frac{1}{\kappa}\left[\frac{\left(f_{b} v_{2}^{s}\right)^{2}}{2}\left(2 \beta^{2}+1+\sqrt{8 \beta^{2}+1+\frac{8 \beta}{f_{b} v_{2}^{s}}}\right)-1\right]^{\frac{1}{2}},
$$

with $\beta=v_{1}^{s} / v_{2}^{s}$.

Critical points are determined by the set of parameters at which two (or more) roots coalesce. This implies that the Jacobian of Eq. (12) is not invertible. Anticipating that the nonergodicity parameters will vanish at the critical point, we find for the stability matrix $S^{c}(\boldsymbol{x})_{i j}=\partial_{x_{j}} J_{i}^{c}(\boldsymbol{x})$ (Jacobian matrix at the critical point)

$$
\begin{aligned}
& S^{c}(\boldsymbol{x})_{1 j}=\delta_{1 j}-\left(V_{\|}^{\mathrm{re}}\right)_{j}, \\
& S^{c}(\boldsymbol{x})_{2 j}=\delta_{2 j}-\left(V_{\|}^{\mathrm{im}}\right)_{j}, \\
& S^{c}(\boldsymbol{x})_{3 j}=\delta_{3 j}-\frac{2 \sum_{i=1}^{3}\left(W_{\perp}\right)_{i j} x_{i}-x_{3}\left(U_{\perp}\right)_{j}}{\sum_{i=1}^{3}\left(U_{\perp}\right)_{i} x_{i}} .
\end{aligned}
$$

This representation makes use of $x_{i} \ll 1$, the symmetry of $W_{\perp}$ and the condition for the root $F_{3}^{c}(\boldsymbol{x})=0$. In classical MCT, the stability matrix is only a function of the coupling coefficients. This allowed it to reformulate the condition of a noninvertible Jacobian into the problem of finding an eigenvector of the stability matrix corresponding to the eigenvalue 0 . In our case here, however, the stability matrix depends additionally on the critical nonergodicity parameter $\boldsymbol{x}$. As a consequence, the linear problem of finding an eigenvector to the eigenvalue 0 of the stability matrix transforms into solving the nonlinear problem $S^{c}(\boldsymbol{h}) \boldsymbol{h}=0$. This avoids the problem of determining the limit $|\boldsymbol{x}| \rightarrow 0$ of the stability matrix $S^{c}(\boldsymbol{x})$, because the representation is scale-free, i.e., any scalar multiple of $\boldsymbol{h}$ is a solution as well. See Appendix B where the critical force [Eq. (13)] is derived following this strategy.

For the analysis of the critical (long-time) dynamics, we expand Eq. (11) for small $s$ and large $\phi_{i}(s)$ and find

$$
\begin{aligned}
\phi_{1}(s)+s\left(\phi_{1}^{2}(s)-\phi_{2}^{2}(s)\right) \approx & \sum_{i=1}^{3}\left(V_{\|}^{\mathrm{re}}\right)_{i} \phi_{i}(s)+\tau_{1}, \\
\phi_{2}(s)+2 s \phi_{1}(s) \phi_{2}(s) \approx \sum_{i=1}^{3}\left(V_{\|}^{\mathrm{im}}\right)_{i} \phi_{i}(s)+\tau_{2}, & (15 \mathrm{a}) \\
\phi_{3}(s)+s \phi_{3}^{2}(s) \approx & \frac{\sum_{i, j=1}^{3}\left(W_{\perp}\right)_{i j} \phi_{i}(s) \phi_{j}(s)}{\sum_{i=1}^{3}\left(U_{\perp}\right)_{i} \phi_{i}(s)} \\
& +\frac{\sum_{i=1}^{3}\left(V_{\perp}\right)_{i} \phi_{i}(s)}{\sum_{i=1}^{3}\left(U_{\perp}\right)_{i} \phi_{i}(s)}+\tau_{3} \\
& -\tau_{3} \frac{\sum_{i, j=1}^{3}\left(W_{\perp}\right)_{i j} \phi_{i}(s) \phi_{j}(s)}{\left[\sum_{i=1}^{3}\left(U_{\perp}\right)_{i} \phi_{i}(s)\right]^{2}} .
\end{aligned}
$$

We can now use the ansatz for an approximate solution of $\phi_{i}(s)$,

$$
\phi_{i}(s)=h_{i} s^{-1 / 2}+c_{i}+O\left(s^{1 / 2}\right)
$$

to derive equations for the unknown parameters $h_{i}$, the so called critical amplitudes. This ansatz implies $s^{2} \phi^{3}(s)=$ $O\left(s^{1 / 2}\right)$ and $\phi^{-1}(s)=O\left(s^{1 / 2}\right)$ and yields in first order

$$
\begin{aligned}
& 0=s^{-1 / 2}\left(h_{1}-\sum_{i=1}^{3}\left(V_{\|}^{\mathrm{re}}\right)_{i} h_{i}\right)+O\left(s^{0}\right), \\
& 0=s^{-1 / 2}\left(h_{2}-\sum_{i=1}^{3}\left(V_{\|}^{\mathrm{im}}\right)_{i} h_{i}\right)+O\left(s^{0}\right), \\
& 0=s^{-1 / 2}\left(h_{3}-\frac{\sum_{i, j=1}^{3}\left(W_{\perp}\right)_{i j} h_{i} h_{j}}{\sum_{i=1}^{3}\left(U_{\perp}\right)_{i} h_{i}}\right)+O\left(s^{0}\right) .
\end{aligned}
$$

Solving this set of equations for arbitrary $s$ up to order $O\left(s^{-1 / 2}\right)$ requires $\boldsymbol{h}$, the vector of critical amplitudes, to fulfill 
the following fixed-point equation:

$$
\begin{aligned}
& h_{1}=\sum_{i=1}^{3}\left(V_{\|}^{\mathrm{re}}\right)_{i} h_{i}, \\
& h_{2}=\sum_{i=1}^{3}\left(V_{\|}^{\mathrm{im}}\right)_{i} h_{i}, \\
& h_{3}=\frac{\sum_{i, j=1}^{3}\left(W_{\perp}\right)_{i j} h_{i} h_{j}}{\sum_{i=1}^{3}\left(U_{\perp}\right)_{i} h_{i}} .
\end{aligned}
$$

This equation is scale free with respect to $\boldsymbol{h}$, since any scalar multiple of $\boldsymbol{h}$ is again a solution. The magnitude of $\boldsymbol{h}$ can be fixed using the higher order terms to determine the vector $c$ introduced in the expansion Eq. (16) as worked out in Chap. 6 of Ref. [25]. The critical exponent of $-1 / 2$ in Laplace space translates into a critical power law in time space with exponent $-1 / 2$ as well. This critical behavior can be verified numerically as shown in Fig. 2. Close to the critical point this power law can be observed over more than six decades in the numerical solutions.

As anticipated above, $\boldsymbol{h}$ is an eigenvector of the critical stability matrix $S^{c}(\boldsymbol{h})$ to the eigenvalue 0 , i.e., $S^{c}(\boldsymbol{h}) \boldsymbol{h}=0$, which can be verified by direct calculation. A Taylor expansion of this solution is possible and leads to a continuous type A transition, which can be confirmed numerically. Inverting Eq. (16) to find the critical behavior in the time domain, we corroborate the resulting $s^{-1 / 2}$ power law numerically, including the prefactors determined by $\boldsymbol{h}$. This is shown in Fig. 2, where we plot the numerical solutions for decreasing but finite distances $|\delta|$ from the critical point. The critical law $\phi_{i}(t)=\alpha h_{i} t^{-1 / 2}$ is shown as black dotted line, where the magnitude $\alpha$ has been adjusted to the data for $i=1$.

In Appendix D we derive a scaling law for the time evolution close to the critical point. It connects the timescale for the deviation from the critical law to the inverse square of the relative distance from the critical force, i.e., $\tau=\delta^{-2}$. This can be verified in the numerical solutions shown in Fig. 2.

To conclude, the beta-scaling analysis of this schematic model results in a continuous transition with a critical power law with exponent $-1 / 2$ in time and Laplace domain. A special point is that the eigenvector equation $S^{c}(\boldsymbol{h}) \boldsymbol{h}=$ 0 for the stability matrix becomes a nonlinear problem. This leads to a scale free fixed-point equation for the critical amplitude $\boldsymbol{h}$.

\section{Beta-scaling analysis of the full model}

For the analysis of the full model, we will interpret the wave vector as a discrete variable [i.e., $\Phi^{s}(t) \in \mathbb{C}^{n}$ ] instead of a continuous one $\left[\Phi^{s}(t): \mathbb{R}^{3} \rightarrow \mathbb{C}\right]$. This is also done for the numerical solution and simplifies some of the arguments. The discrete wave vectors will be labeled by the index $q$. As a consequence, the equations of motion become a finite dimensional system of equations and the primitive memory functionals can be expressed as matrices. As before, we use the constant-bath approximation $\Phi_{q}(t) \rightarrow f_{q}$ so that we can rewrite the equations of motion as follows (the argument $s$ is
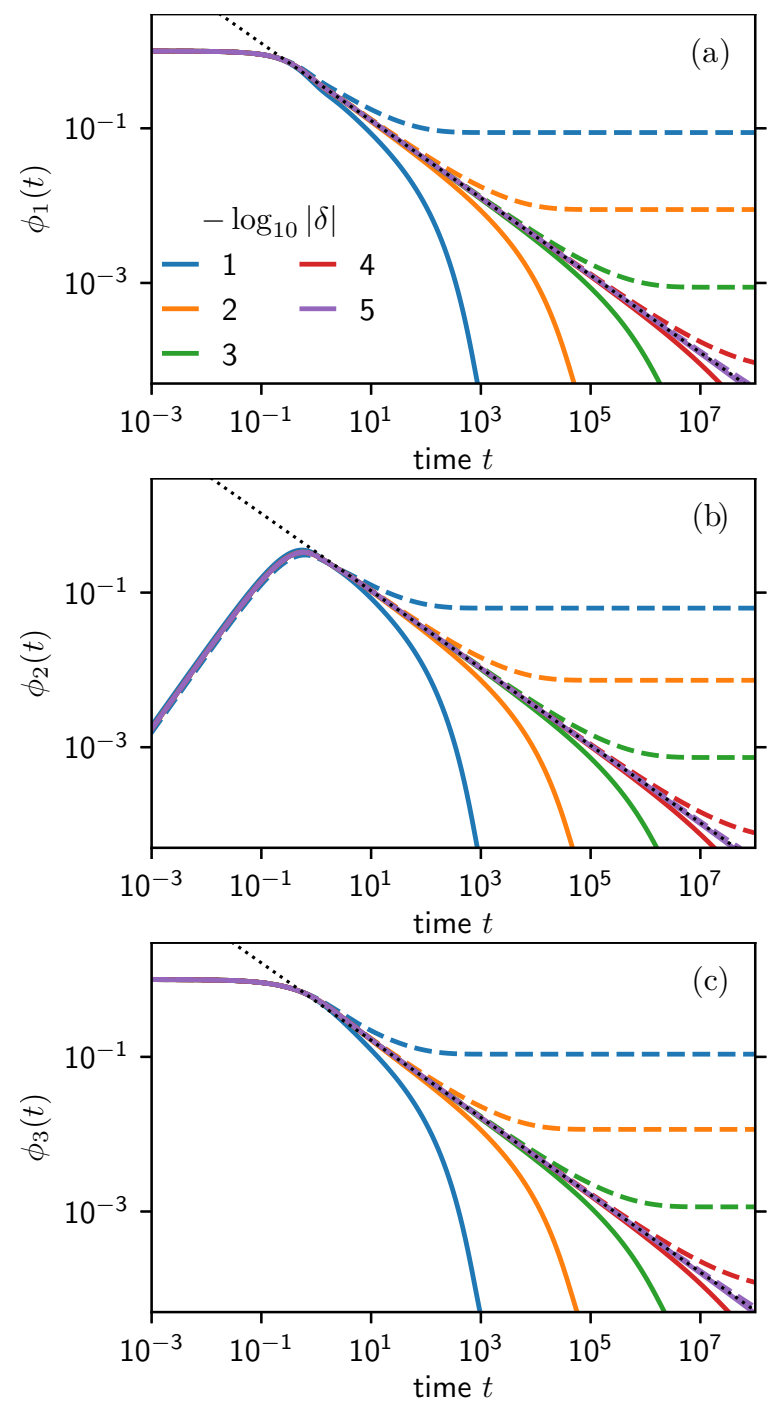

FIG. 2. Critical behavior of the schematic model. Correlation functions for $\epsilon=0.1, v_{1}^{s}=v_{2}^{s}=2, \kappa_{\|}=\kappa_{\perp}=1$, constant bath and different distances $\delta=\left(F_{\text {ext }}-F_{c}\right) / F_{c}$ to the critical force as labeled. Dashed lines indicate $\delta<0$, while solid lines indicate $\delta>0$. The critical law $\phi_{i}^{c}(t)=\alpha h_{i} t^{-1 / 2}$ is shown as dotted line. The critical amplitude $h_{i}$ is a solution of Eq. (18) and its magnitude $\alpha$ is adjusted based on the data in the top panel. The line color and style is the same across all panels.

skipped to keep the equation concise):

$$
\begin{aligned}
0= & \left(s \tilde{\Phi}_{q}^{s}-1\right)+\frac{1}{s}\left[s \tilde{\Phi}_{q}^{s} \Gamma_{q}+\left(s \tilde{\Phi}_{q}^{s}-1\right) C_{q}^{T} s \tilde{\boldsymbol{\Phi}}^{s}\right] \\
& +\frac{1}{s^{2}}\left[\left(s \tilde{\Phi}_{q}^{s}-1\right)\left(s \tilde{\boldsymbol{\Phi}}^{s}\right)^{T} A_{q}\left(s \tilde{\boldsymbol{\Phi}}^{s}\right)+s \tilde{\Phi}_{q}^{s} B_{q}^{T}\left(s \tilde{\boldsymbol{\Phi}}^{s}\right)\right],
\end{aligned}
$$

with the abbreviations

$$
\begin{aligned}
\boldsymbol{v}^{T} A_{q} \boldsymbol{w}= & \frac{1}{2}\left[\mu_{q}^{x x}(\boldsymbol{v}) \mu_{q}^{z z}(\boldsymbol{w})+\mu_{q}^{x x}(\boldsymbol{w}) \mu_{q}^{z z}(\boldsymbol{v})\right] \\
& -\left[\mu_{q}^{x z}(\boldsymbol{v}) \mu_{q}^{x z}(\boldsymbol{w})\right] \\
B_{q}^{T} \boldsymbol{v}= & \mu_{q}^{x x}(\boldsymbol{v}) q_{z}^{2}+\mu_{q}^{x z}(\boldsymbol{v})\left(-2 q_{x} q_{z}\right)+\mu_{q}^{z z}(\boldsymbol{v}) q_{x}^{2} \\
& +i F_{\mathrm{ext}}\left[-\mu_{q}^{x x}(\boldsymbol{v}) q_{z}+\mu_{q}^{x z}(\boldsymbol{v}) q_{x}\right]
\end{aligned}
$$




$$
\begin{aligned}
C_{q}^{T} \boldsymbol{v} & =\mu_{q}^{x x}(\boldsymbol{v})+\mu_{q}^{z z}(\boldsymbol{v}), \\
\Gamma_{q} & =q_{x}^{2}+q_{z}^{2}-i F_{\mathrm{ext}} q_{z},
\end{aligned}
$$

using matrix-vector multiplications with auxiliary vectors $v$ and $\boldsymbol{w}$. We use the boldface symbol $\tilde{\boldsymbol{\Phi}}^{s}=\left(\tilde{\Phi}_{q}^{s}\right)_{q}$ to indicate the vector of all correlation functions $\tilde{\Phi}_{q}^{s}$ for different wave vectors $q$. From Eq. (6) we obtain the long-time-limits of the memory functionals via $\mu_{q}^{\alpha \beta}(\boldsymbol{v}):=m_{q}^{\alpha \beta}[\boldsymbol{v}, \boldsymbol{f}]$ with the bath nonergodicity parameter $f:=\lim _{t \rightarrow \infty} \phi(t) . A_{q}$ is a matrix with constant coefficients, $B_{q}$ and $C_{q}$ are vectors with constant coefficients and $\Gamma_{q}$ is a scalar for each wave vector, which is labeled by the index $q$. Notice that only $B_{q}^{T}$ and $\Gamma_{q}$ depend on the external force. To find the proper long time behavior it is convenient to introduce the $S$-transform as $s$ times the Laplace-transform ( $s$ being the variable in Laplace space). This has the advantage that the power-laws in the timedomain and for the $s$-transform are simply reciprocals of each other and constant functions are transformed into constant functions. This rationalizes the notation $s^{-1}\left(s \tilde{\Phi}_{q}^{s}\right)$ instead of writing $\tilde{\Phi}_{q}^{s}$ only.

As above, we can find the determining equation for the nonergodicity parameters $f_{q}^{s}:=\lim _{t \rightarrow \infty} \phi_{q}^{s}(t)=$ $\lim _{s \rightarrow 0} s \tilde{\Phi}_{q}^{s}(s)$ by multiplying with $s^{2}$ and taking the limit $s \rightarrow 0$. It reads

$$
0=\left(f_{q}^{s}-1\right)\left[\left(\boldsymbol{f}^{s}\right)^{T} A_{q} \boldsymbol{f}^{s}\right]+f_{q}^{s}\left(B_{q}^{T} \boldsymbol{f}^{s}\right) .
$$

The corresponding Jacobian is given by

$$
\begin{aligned}
S_{q p}= & \delta_{q p}\left[\left(\boldsymbol{f}^{s}\right)^{T} A_{q} \boldsymbol{f}^{s}+B_{q}^{T} \boldsymbol{f}^{s}\right]+f_{q}^{s} B_{q}^{T} \hat{\boldsymbol{p}} \\
& +\left(f_{q}^{s}-1\right)\left[\left(\boldsymbol{f}^{s}\right)^{T} A_{q} \hat{\boldsymbol{p}}+(\hat{\boldsymbol{p}})^{T} A_{q} \boldsymbol{f}^{s}\right],
\end{aligned}
$$

where $\hat{\boldsymbol{p}}$ is the unit vector in $p$-direction. This matrix is zero in the limit $\boldsymbol{f}^{s} \rightarrow 0$.

Equation (21) can be solved for $f_{q}^{s} /\left(1-f_{q}^{s}\right)$ to have the same form as in classical MCT. Then, the right hand side corresponds to the memory functional, which is always a polynomial in classical MCT. In our case, the resulting right hand is a quotient of the memory functionals $\left(\boldsymbol{f}^{s}\right)^{T} A_{q} \boldsymbol{f}^{S}$ and $B_{q}^{T} \boldsymbol{f}^{s}$. This poses a challenge for taking the limit $\left|f_{q}^{s}\right| \rightarrow 0$, as the limiting value of the quotient will depend on the details of how the different values of $f_{q}^{s}$ approach zero.

It is also possible to rewrite Eq. (21) in an alternative form as a fixed-point problem,

$$
0=f_{q}^{s}-\frac{\left(\boldsymbol{f}^{s}\right)^{T} A_{q} \boldsymbol{f}^{s}}{B_{q}^{T} \boldsymbol{f}^{s}+\left(\boldsymbol{f}^{s}\right)^{T} A_{q} \boldsymbol{f}^{s}} .
$$

Since $\boldsymbol{f}^{s}$ is complex valued, the calculation of the Jacobian becomes a rather tedious task, as real and imaginary parts have to be treated as separate variables (see Appendix E or Eqs. (5.37)- (5.41) in Chap. 5.4 of Ref. [25]). It turns out that the Jacobian which is constructed with this knowledge from Eq. (23) behaves like the stability matrix in classical MCT, as one of its eigenvalues approaches 0 at the delocalization transition. Similar to the schematic calculation, it is possible to derive a scale-free fixed point iteration for the corresponding eigenvector, the so called critical amplitude (see Eq. (E8) and Chap. 5.4 of Ref. [25] for the details of this tedious calculation). While the critical amplitude remains finite for small
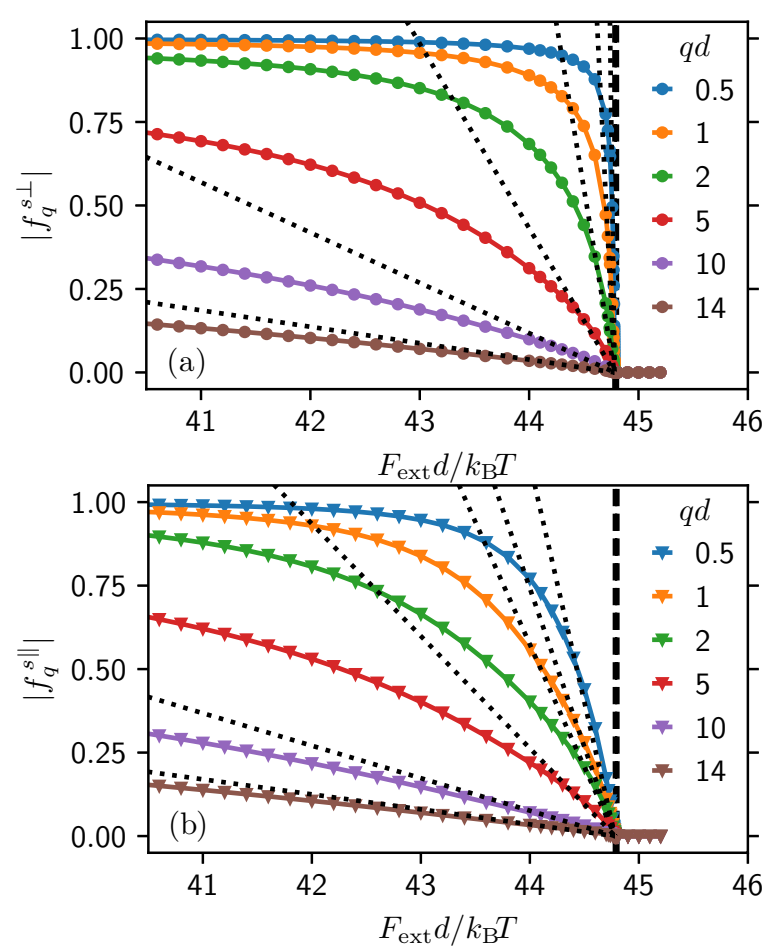

FIG. 3. Nonergodicity parameters $\left|f_{\boldsymbol{q}}^{s}\right|=\lim _{t \rightarrow \infty}\left|\phi_{q}^{s}(t)\right|$ for perpendicular (top panel) and parallel (bottom panel) wave vectors with $q=0.5,1,2,5,10,14$ from top to bottom. The dashed line indicates the critical force and the dotted lines the asymptotic behavior close to the critical force. These data were obtained with the model with $N_{\log }=10$ points. On this scale there is no difference to the other model with $N_{\log }=25$.

wave vectors in classical MCT, (cf. Fig. 4.6 in Ref. [24]), we find that in our model the critical amplitude diverges for small wave vectors: like $q_{x}^{-2}$ in the perpendicular direction and like $q_{z}^{-1}$ for the parallel direction (see inset in the lower panel of Fig. 6). This renders it impossible to normalize the critical amplitude by its norm. Instead, we have to fix the value of the critical amplitude at a certain wave vector.

This divergence can be understood by checking the implications of the critical amplitude. For example, it determines the scaling of the nonergodicity parameters close to the critical point as shown in Fig. 3. For a type A transition, we have the following asymptotic expansion for $\delta:=\left(F_{\text {ext }}-F_{c}\right) / F_{c}<0$,

$$
f_{q}^{s}=-h_{q} \delta+O\left(\delta^{2}\right),
$$

with an appropriate scaling of $h_{q}$. The dashed lines in Fig. 3 are given by this relationship and the values of $h_{q}$ are shown in the lower panel of Fig. 6. The scaling factor for $h_{q}$ is set at the largest wave vector in the perpendicular direction. We notice that the decay of the nonergodicity parameter becomes steeper and steeper for smaller wave vectors. This effect is more prominent for wave vectors perpendicular to the force direction as was the divergence of the critical amplitude.

For the analysis of the critical dynamics, we start out with Eq. (19) and focus on the long time behavior around the critical force. Formal calculations show that we can expect a $s^{-1 / 2}$ or a $t^{-1 / 2}$ power-law scaling of the correlation functions at the delocalization transition (see Chap. 5.6 in Ref. [25] for 
the details). These findings can also be confirmed numerically. We note, however, that the onset of this scaling depends on the wave-vector magnitude. The smaller the magnitude, the later (longer times or smaller Laplace frequencies) the onset of the critical power law.

This finding also disproves the existence of a master curve for the correlation functions for all $q$ at a finite distance from the critical point, which is usually assumed for the beta-scaling analysis. Nevertheless, we find a master curve for the primitive memory kernels, i.e., a factorization of the Laplace frequency and wave-vector dependency. With this information, we can work out the critical power laws for the moments, which can be traced back to the evaluation of a combination of primitive memory functionals (see Chap. 5.8 of Ref. [25] for details). We find $\langle z\rangle \propto t^{-\alpha},\left\langle(x-\langle x\rangle)^{2}\right\rangle \propto$ $t^{-\alpha}$ and $\left\langle(z-\langle z\rangle)^{2}\right\rangle \propto t^{-2 \alpha}$ using a general critical power law with exponent $\alpha$ [i.e., $\Phi_{q}^{s}(s) \propto s^{\alpha}$ ]. With the choice $\alpha=$ $-1 / 2$, based on the results from the schematic model, the exponents above reduce to $1 / 2,1 / 2$ and 1 .

For the behavior close to the critical force, we can identify a timescale, which scales like $\delta^{-2}$ as can be observed in Fig. 9. This is the same scaling as derived for the schematic model as discussed in the previous section. This highlights again that the schematic model represents the critical features of the full model.

With the knowledge of the scaling of the memory functionals, we can also discuss the scaling of the stationary velocity as function of the distance to the critical force. The stationary velocity $v_{\mathrm{st}}:=\lim _{t \rightarrow \infty} \partial_{t}\langle z\rangle(t)$ is related to the integral over the memory functional $m_{0}^{z z}(t):=\lim _{q \rightarrow 0} m_{q}^{z z}(t)$ via

$$
v_{\mathrm{st}}=\frac{F_{\mathrm{ext}}}{1+\int_{0}^{\infty} m_{0}^{z z}\left(t^{\prime}\right) d t^{\prime}}
$$

(see Eq. (2.189) in Refs. [25] and [21]). Below and at the critical force, this memory integral diverges, leading to a vanishing stationary velocity. Above the critical force, this integral becomes finite as the memory functional decays on a certain timescale. A scaling argument (see Sec. 5.6.3 of Ref. [25] and Appendix D) suggests $\int_{0}^{\infty} m_{0}^{z z}\left(t^{\prime}\right) d t^{\prime} \propto \delta^{-1}$, which results in $v_{\text {st }} \propto \delta$ to first order for $\delta>0$.

Summarizing the beta-scaling analysis for the full model, we find for the given discretization a type A transition with a critical power law with exponent $-1 / 2$ for the correlation functions. The critical amplitude can be determined via a scale-free fixed-point-equation and has diverging values in the limit $q \rightarrow 0$. The critical power law is most pronounced for large wave numbers, while being shadowed for small wave numbers. The mean displacement and the variances perpendicular and parallel to the force direction exhibit critical power laws as well with exponents $1 / 2,1 / 2$ and 1 , respectively.

\section{SIMULATIONS}

A polydisperse system of quasihard spherical particles is simulated with Langevin dynamics. The equation of motion for particle $j$ is given by [29]

$$
m \ddot{\boldsymbol{r}}_{j}=-\gamma_{0} \dot{\boldsymbol{r}}_{j}+\boldsymbol{\eta}_{j}(t)+\sum_{i} \boldsymbol{F}_{i j}+\delta_{j 1} \boldsymbol{F}_{\mathrm{ext}}
$$

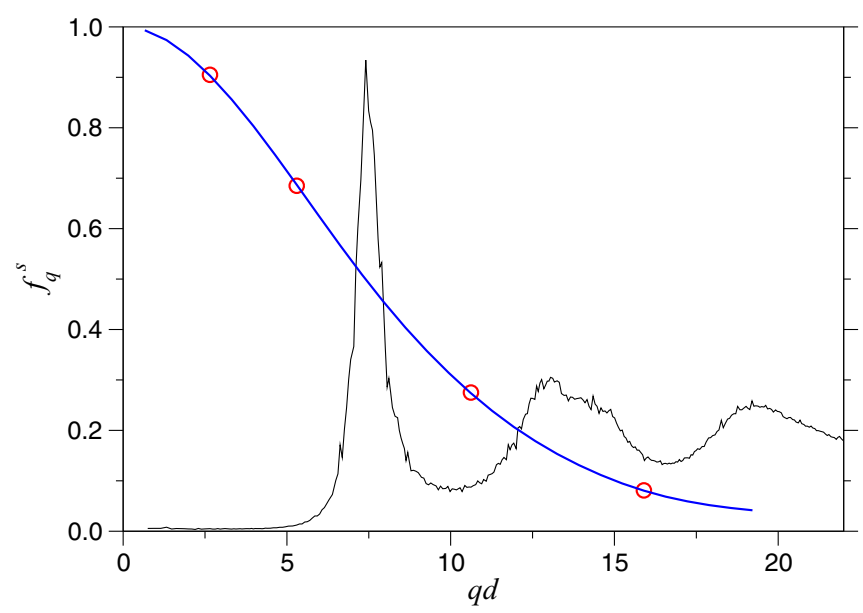

FIG. 4. Nonergodicity parameter and structure factor of the glassy host system. The red circles identify some typical wave-vector moduli studied below.

where the friction with the solvent, $\gamma_{0} \dot{\boldsymbol{r}_{j}}$, is given by the coefficient $\gamma_{0}$, and $\boldsymbol{\eta}_{j}(t)$ is a random force linked to the friction coefficient via the fluctuation-dissipation theorem [29]. The particle-particle interactions, $\boldsymbol{F}_{i j}$, is given in our case by the inverse-power potential:

$$
V\left(d_{i j}\right)=k_{\mathrm{B}} T\left(d / d_{i j}\right)^{-36}
$$

with $d_{i j}$ the center to center distance between particles. Finally, the external force $\boldsymbol{F}_{\text {ext }}$ is applied only to the tracer, labeled as $j=1$.

The simulated glass has a volume fraction, $\varphi=0.62$, calculated considering hard spheres of diameter $d$. The glass transition for this system has been estimated previously by an MCT analysis yielding $\varphi_{g}=0.596$ [30,31]. Hence, this system is as far from the glass transition as the system used for the theoretical calculations. The preparation and properties of this glass have been discussed previously [22,32]. In essence, the system is equilibrated in a fluid state with the same density and moderate attractions; the attractions are then suddenly removed, leaving only the repulsive interactions. The system is then aged for a long time. The dynamics of the aged glass does not show any sign of further evolution for the same time range used in the study of microrheology presented here. Fig. 4 presents the nonergodicity parameter of the glass and the structure factor, for reference. Further details of the preparation procedure can be found in Refs. [22,32].

In our simulations of microrheology we focus on the transient regime. A particle is randomly selected as tracer and at $t=0$, a constant external force is applied to it, pulling it through the system. The tracer trajectory is monitored as a function of time. The tracer is allowed to travel through the simulation box more than once, as we could not identify any different behavior between the first and consecutive passages. The results presented below are the average over ca. 5000 independent trajectories (tracers) for every force. For $F_{\text {ext }}=$ $80 k_{\mathrm{B}} T / d$, which we identify as the critical force, 25000 trajectories have been simulated. The maximum time in the simulations after equilibration is $t D_{0} / d^{2}=25$. 
The main observable of interest is the tracer position correlation function, as discussed in the theory section, namely, the Fourier transform of the tracer displacement probability distribution. In the simulations, the wave vector is restricted due to the periodic boundary conditions. Even more, since the dynamics of the tracer depends on the direction, only wave vectors with moduli multiple of $2 \pi / L$, are allowed in the force direction, with $L$ the dimension of the simulation box in this direction. Some of the wave vectors studied below (in particular in Fig. 7) are shown as red circles in Fig. 4.

In the simulations we consider $N=1000$ particles in a cubic box with periodic boundary conditions. The box size is set by the volume fraction of the particles, $\phi=0.62$, yielding $L=9.48 d$, with $d$ the average particle diameter. All particles have the same mass, $m$; diameters are distributed according to a flat distribution of width $\Delta=0.1 d$, to avoid crystallization. In the simulations we set to $m=1, d=1$ and the thermal energy is $k_{\mathrm{B}} T=1$. The solvent friction coefficient is set to $\gamma_{0}=10 \sqrt{m k_{\mathrm{B}} T} / d$, giving a diffusion coefficient of the free particle $D_{0}=k_{\mathrm{B}} T / \gamma_{0}=0.1 d \sqrt{k_{\mathrm{B}} T / m}$. Time is measured in units of the Brownian time $\tau_{B}=d^{2} / D_{0}$. The equations of motion are integrated using a Heun algorithm [33] with a time step of $0.00025 d \sqrt{m / k_{\mathrm{B}} T}=2.5 \times 10^{-5} \tau_{B}$.

\section{RESULTS AND DISCUSSIONS}

The theoretical predictions for the critical force in the section about the bifurcation analysis were based on the evaluation of the long-time limit of the correlation functions. This limit is, however, unreachable in the simulations, where a finite-time analysis must be performed. We will thus first study if the critical force and amplitude can be properly estimated from a finite time analysis. Then, the power-law behavior of the correlation function and tracer displacement at the critical force, predicted by our model, are tested with simulations. Finally, we will show that the stationary velocity for a long but finite time provides an additional estimation of the critical force. These results will provide three different methods of determining the critical force and the properties of the transition, corroborated by our simulations, that can be used in experimental systems.

\section{A. Estimation of the critical force}

In this section, we will analyze a procedure to estimate the critical force based on finite time results. While the critical force is defined based on the vanishing of the nonergodicity parameter (the long-time-limit of the tracer position correlation function), this cannot be achieved in simulations. Additionally, the MCT glass transition appears as crossover in experimental systems or simulations, which leads to finite relaxation times of the bath [34]. This very slow structural relaxation is neglected in the theory, yet contained in the simulations and anticipated the following analysis. Therefore, we model the procedure for the simulations by evaluating the theoretical result for the tracer position correlation function at a large but finite time as an approximation to the nonergodicity parameter. This is done by solving the model described by Eqs. (2), (5), and (6) using the methods described in Sec. II B and Refs. [22,25]. From these values, the critical force and
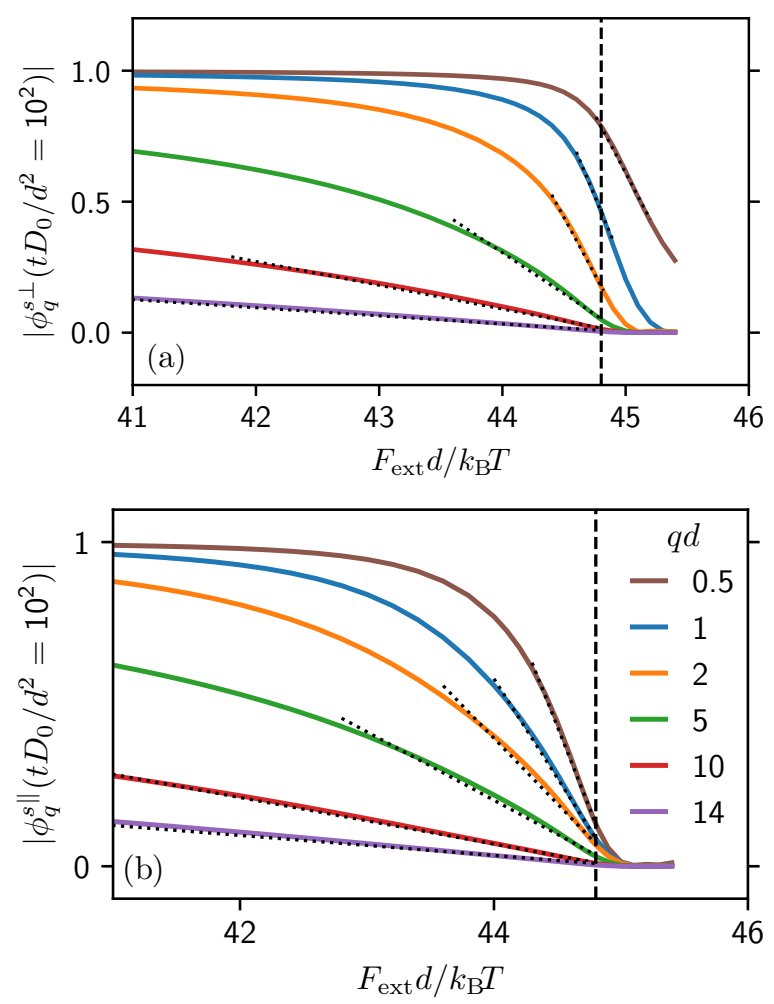

FIG. 5. Values of the tracer position correlation function for $t=10^{2} d^{2} / D_{0}$ as a function of the external forces for different wave vectors perpendicular to the external force (upper panel) and parallel to it (lower panel). Numerical solutions of the MCT equations are shown. The dashed vertical line indicates the true critical force. The dotted black lines indicate linear fits to the nonergodicity parameters to determine the critical force shown in Fig. 6.

amplitude are estimated, and compared with the values obtained from the true long time limit.

The values of the theoretical correlation functions as a function of the external force for different wave vectors at the finite time $t D_{0} / d^{2}=10^{2}$ are shown in Fig. 5. Wave vectors parallel and perpendicular to the force direction are presented; since the correlation functions in the force direction are complex, the modulus is studied. While the type A behavior for large wave vectors is very similar to the results for the true long-time limit (shown in Fig. 3), there are some differences for small wave vectors. This graph shows that the diverging slope of $f_{q}$ as a function of the external force at the critical force value is replaced by a finite slope, which intersects with 0 at larger forces. This will induce systematic errors in the estimation of the critical force value when analyzing small wave vectors. Although this effect is noticed for both directions, the transversal one shows a more dramatic effect, consistent with the theoretical analysis for the critical amplitude.

The analysis of the correlation function at finite times as a function of the external force is presented in Fig. 6. Here, the value of the critical force (upper panel) is extracted from the intersection of the linear fit of $f_{q}$ vs $F_{\text {ext }}$ with the $x$ axis. The range of the fit has been adjusted to capture the linear behavior best. The errorbars indicate the uncertainty from the fit. As mentioned previously, the critical force for small wave vectors is overestimated, more prominently when the wave 

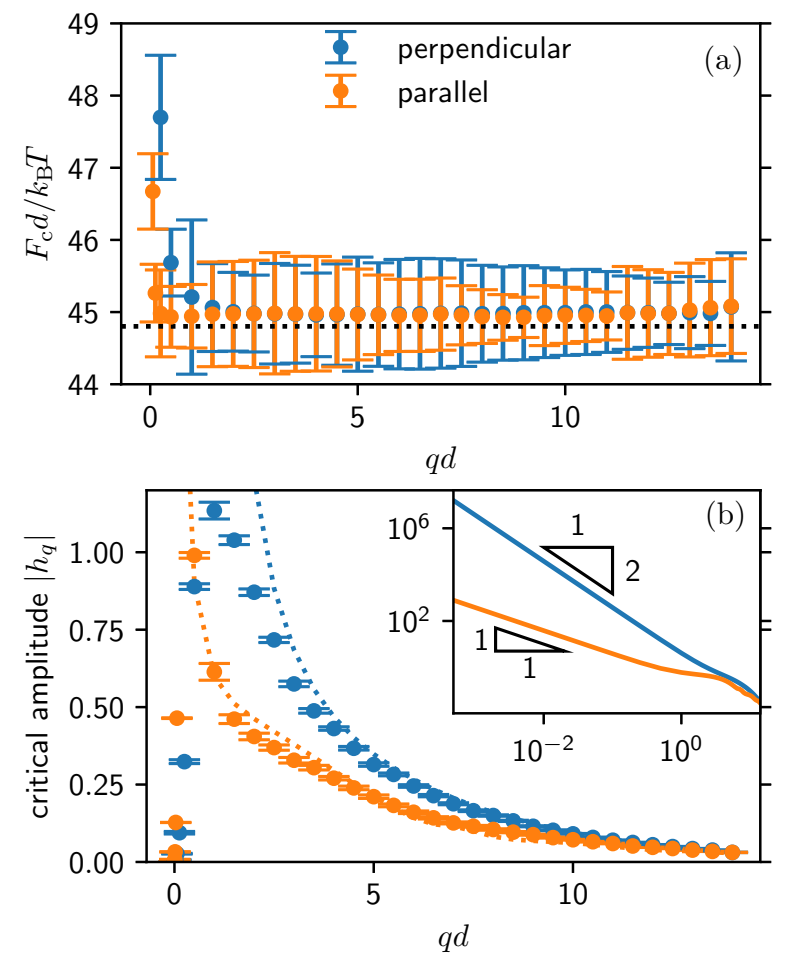

FIG. 6. Critical force (upper panel) and critical amplitude (lower panel) obtained from time-dependent MCT calculations as a function of the wave vector. Symbols show data estimated from the linear fittings of $f_{c}$ vs $F_{\text {ext }}$ for finite times (see Fig. 5). Errorbars indicate the uncertainties in these parameters arising from the fit. The horizontal dashed line in the upper panel marks the true critical force and the dashed lines in the lower panel are the critical amplitudes obtained in the limit $t \rightarrow \infty$ of $\phi_{q}^{s}(t)$. The inset shows the same critical amplitudes on a log-log scale establishing the $\left(q_{x}\right)^{-2}$ and $\left(q_{z}\right)^{-1}$ divergences in the low $q$ limit.

vector is perpendicular to the external force. However, for not-too-small wave vectors, the estimate is independent of $\boldsymbol{q}$ (direction or modulus), and more importantly, agrees with the value extracted from the analysis of the long-time limit.

The critical amplitude (lower panel) at the finite time is given by the slope of this fit (symbols). Since we have complex valued correlators, we fit real and imaginary part separately and show the modulus of the resulting complex critical amplitude at finite times. It shows stronger deviations from the true critical amplitude (dashed lines), which disappear only for large wave vectors. Both data for the longitudinal and transversal wave vectors display a maximum as a function of $q$, which is not observed in the analysis of the proper long time limit. Looking back at Fig. 5, we realize that this behavior arises from the fact that the correlation functions have not yet decayed to zero above the critical force at finite times. This implies that for small wave vectors, particularly when $\boldsymbol{q}$ is perpendicular to the force, the proper critical amplitude is underestimated.

With these differences in mind, we now turn to the simulation data, and analyze the tracer position correlation functions averaged over time in the range $[10,25] d^{2} / D_{0}$. It must be remembered that the wave vector in the analysis has to be
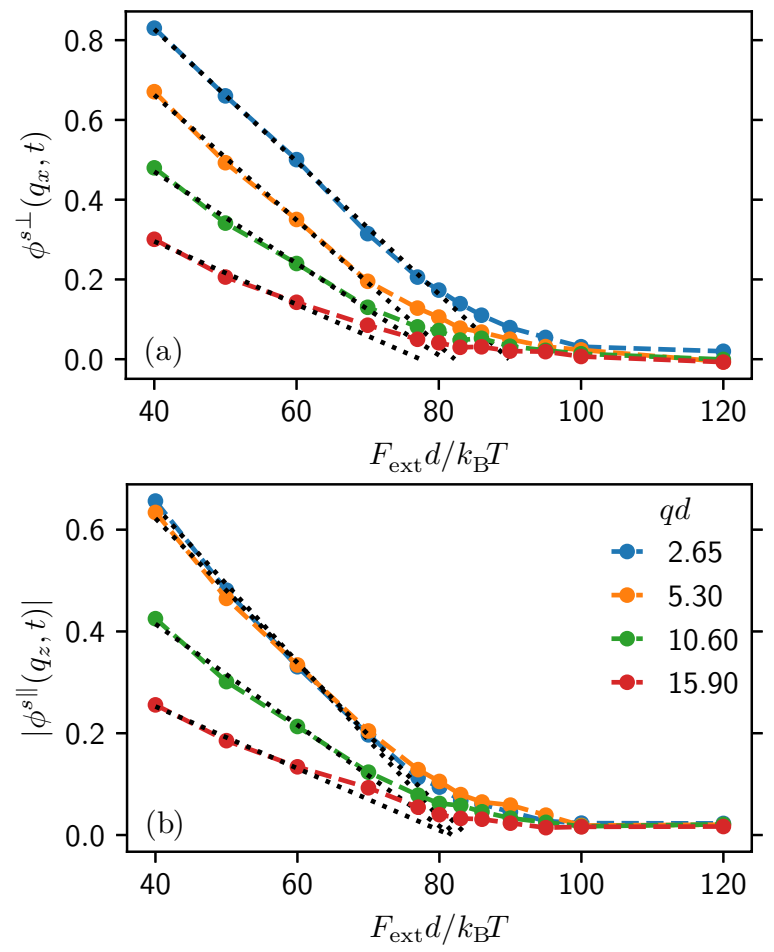

FIG. 7. Value of the tracer position correlation function from simulations for different wave vectors perpendicular to the external force (upper panel) and parallel to it (lower panel) averaged over the time interval $t D_{0} / d^{2} \in[10,25]$. The dotted lines show the linear fits, which are used to estimate the critical force and critical amplitude as shown in Fig. 8. The wave-vector moduli are given in the legend, and are marked by the circles in Fig. 4.

compatible with the periodic boundary conditions. Figure 7 presents the value of the correlation function as a function of the external force for different wave vectors (similar to Fig. 5, wave vectors parallel and perpendicular to the force are considered). As predicted by the theory, the correlation function is lower for wave vectors parallel to the external force. The anomalies reported within the theory for small wave vectors are not observed here, because these wave vectors are not accessible in the simulations.

The values of these correlation functions are fitted with a linear model, following the theoretical prediction. The resulting critical force (from the intercept of the fitting with the $x$ axis) and critical amplitude (slope of the fitting) are shown in Fig. 8. The critical force does not depend on the wave-vector modulus or direction, within the error bars, for large wave vectors, but increases when it is estimated from small wave vectors, in agreement with the theoretical results in Fig. 6. Averaging those results for $2.5<q d<12.5$, we obtain a critical force of $(83.1 \pm 5.8) k_{\mathrm{B}} T / d$ from the parallel direction and $(81.5 \pm 5.8) k_{\mathrm{B}} T / d$ for the perpendicular direction. Given the theoretical analysis presented above, we therefore estimate from these results that the critical force in the simulations is about $F_{c}=80 k_{\mathrm{B}} T / d$, taking into account that the finite time analysis overestimates the critical force. This value is significantly higher than in the theory $F_{c}=44.79 k_{\mathrm{B}} T / d$, although the glass in the theory is ideal, in contrast with the real glass in the simulations. 

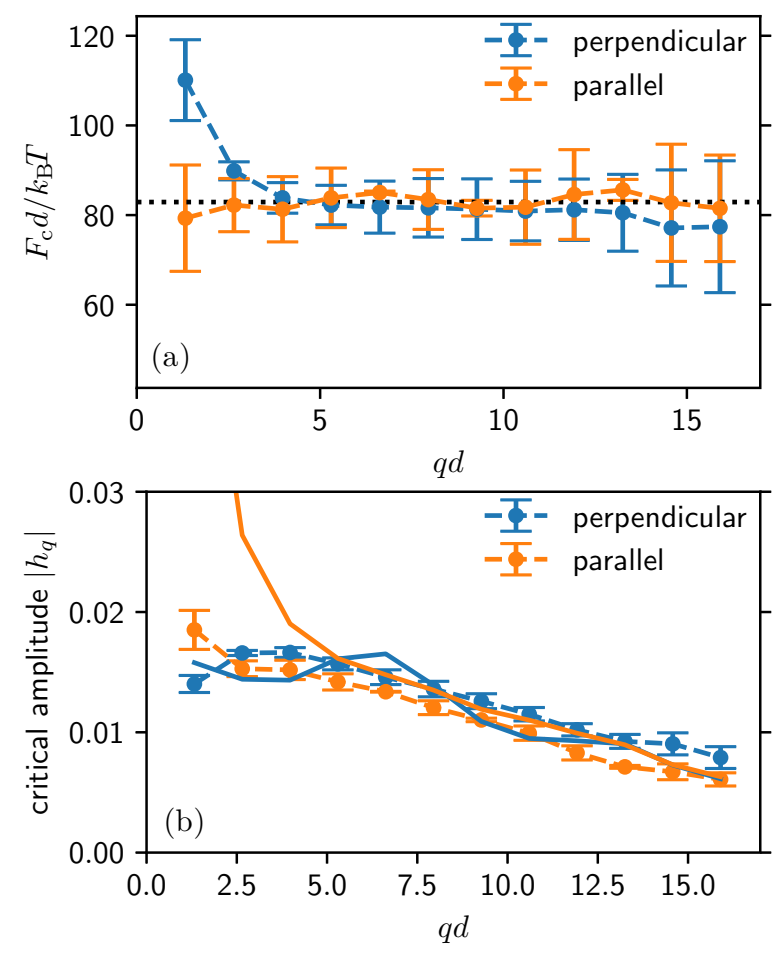

FIG. 8. Critical force (upper panel) and critical amplitude (lower panel) from the simulations for wave vectors parallel and perpendicular to the external force as a function of the modulus of the wave vector. Symbols show data from the linear fitting of $f_{c}$ vs $F_{\text {ext }}$ (see Fig. 7). The dashed horizontal line in the top panel indicates the average critical force for $q d>2.5$ of $82.4 k_{\mathrm{B}} T / d$. The solid lines in the lower panel are estimates of the critical amplitude from the long time behavior of the tracer position correlation function for $F_{\text {ext }}=80 k_{\mathrm{B}} T / d$ (see text below).

The critical amplitude, plotted in the lower panel of Fig. 8, decreases in qualitative agreement with the theoretical results. For small wave vectors, it shows a maximum when the wave vector is perpendicular to the external force, also in agreement with the theoretical results of Fig. 6. However, other details of the theoretical analysis are absent in the simulations; in particular, the critical amplitude does not become independent on the wave-vector direction, and when the wave vector is parallel to the external force the simulation data does not show a maximum.

When the wave vector is parallel to the external force, it must be recalled that the tracer position correlation function is complex valued (thus, the modulus of the correlation function has been studied) [11,22]. For increasing force, the real part of the nonergodicity parameter becomes negative, while the imaginary part describes a maximum. Both components tend linearly to zero at the critical force; the modulus is dominated by the imaginary part. The analysis of the correlation functions at a finite time (not shown) yield the same conclusions as drawn previously. The comparison with the simulations, however, is more difficult due to the large noise-to-signal ratio in the real part.

Summarizing this section, we can identify the critical force consistently from linear extrapolation of the tracer position correlation function at long, but finite times in theory as well as in simulations. The uncertainty for the values of the critical force is largest for small wave vectors due to their strong variation around the critical force. We can also extract the critical amplitudes, which increase with decreasing wave vector for both simulations and theory.

\section{B. Long time behavior of the tracer position correlation function}

We analyze in more detail the tracer position correlation functions in the vicinity of the critical force estimated previously. The theory predicts a power law decay with exponent $-1 / 2$. The onset of this power law depends on the wave vector and its prefactor is described by the critical amplitude for large wave vectors. For a better resolution of the critical force, we use for this section $N_{\log }=25$ points in the nonuniform part of the grid for the theoretical calculations as described in the section about the numerical details.

Figure 9 presents rectification plots of the modulus of the tracer position correlation function with the predicted behavior. For a rectification plot we multiply the correlation functions with the inverse of the anticipated power law. This leads to a straight horizontal line as long as this power law is fulfilled, hence a rectification of the curve. While forces well above or below the critical one do not show the power-law decay, forces close to that indeed follow the expected trend, for all wave vectors moduli and directions. Note that the start of this power-law behavior occurs for earlier times the larger the wave vectors are. It should be also stressed that the trend is also followed even for small wave vectors, despite the difficulties in handling low wave vectors in the theory. Noteworthy, the critical behavior is more difficult to be observed for small wave vectors perpendicular to the external force.

As discussed previously, when the wave vector is parallel to the external force the tracer position correlation function is complex valued. The real part takes on negative values for large wave vectors and tends to zero from below, while the imaginary part is always positive and describes a maximum. Both components indeed exhibit the $t^{-1 / 2}$ decaying to zero (not shown).

The same rectification plots for the simulation data are shown in Fig. 10. In fact, the critical force identified by our previous analysis, $F_{\text {ext }} \approx 80 k_{\mathrm{B}} T / d$, shows a plateau, except for the lowest wave vector studied in the perpendicular direction. In agreement with the theoretical expectations, forces well below the critical force deviate upwards, while forces above it deviate downwards. Also, it is observed that the critical behavior is reached for longer times the smaller the wave vector. This justifies the absence of a power-law behavior in the top-left panel.

Note that the observation of a power-law decay in the tracer position correlation function at long times for all wave-vector moduli and directions can be used as an alternative criterion to identify the critical force. As observed in Fig. 10, this yields the same result as the fitting of the nonergodicity parameters with the external force, $F_{c} \approx 80 k_{\mathrm{B}} T / d$. However, because the observation of a specific behavior in the correlation function is arguable, the extrapolation of the nonergodicity parameters appears as a more robust method.

The prefactor of the $t^{-1 / 2}$ behavior, given by the value of the long time plateaus in Figs. 9 and 10, is the critical 


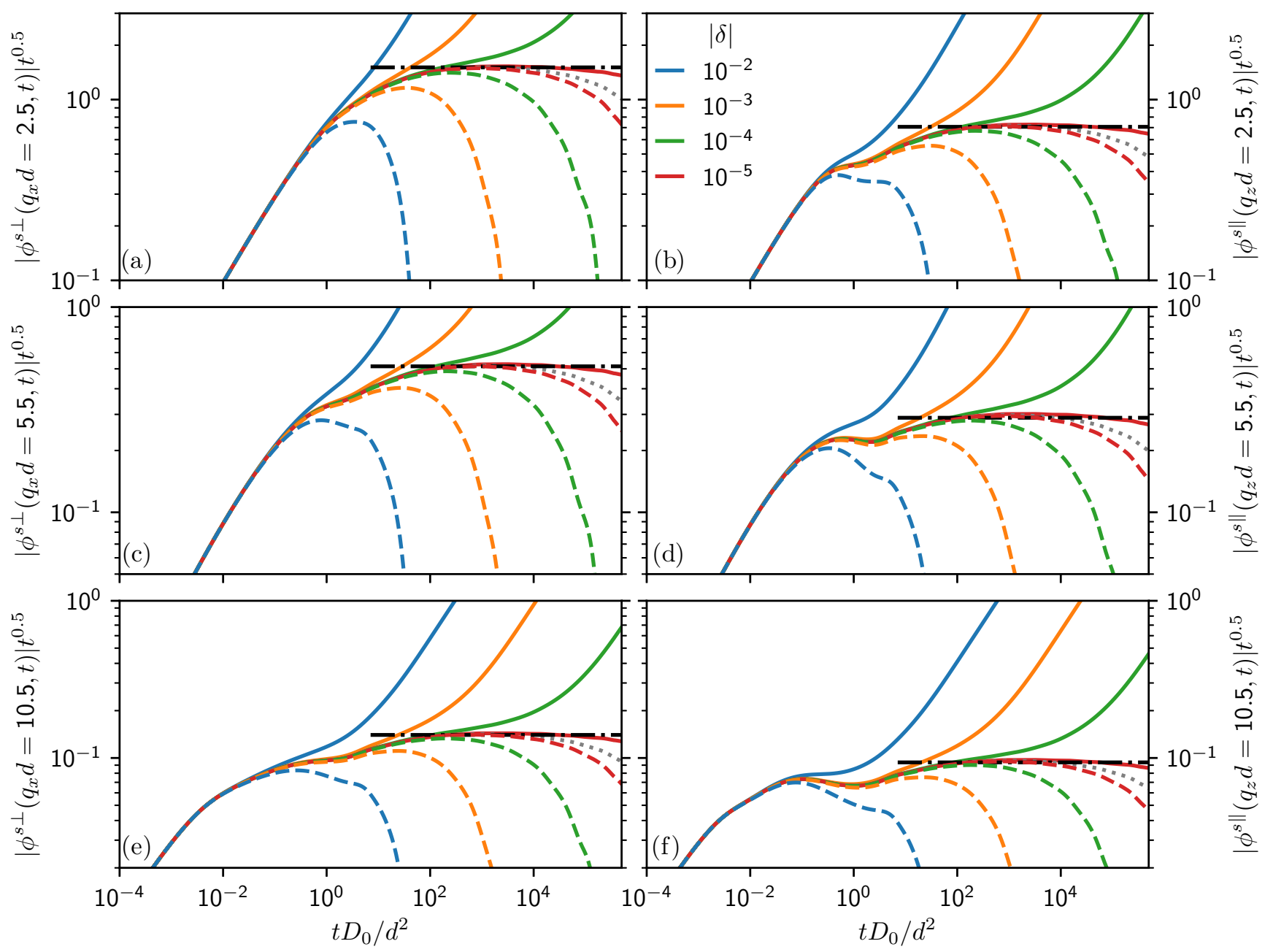

FIG. 9. Rectification plots for the tracer position correlation function for wave vectors perpendicular (left panels) and parallel to the external force (right panels) from the theory. Several forces around the critical one $\left[\delta=\left(F_{\mathrm{ext}}-F_{c}\right) / F_{c}\right]$ are presented. Dashed lines indicate forces above the critical force, solid lines forces below. The dotted line indicates the critical behavior. Different wave vectors are presented: $q d=2.5$ (top panels), $q d=5.5$ (intermediate panels), and $q d=10.5$ (bottom panels).

amplitude, up to a constant factor arising from a microscopic timescale. According to the theory, this should agree with the slope of the nonergodicity vs. the external force (up to this constant factor). The critical amplitude for the theory, is plotted in the lower panel of Fig. 6 as dashed lines; to avoid the ambiguity of the microscopic timescale, both sets are matched at the highest wave vector in the perpendicular direction. At large wave vectors, both estimations of the critical amplitude decay monotonously, and agree for the highest wave vectors. However, differences appear for small wave vectors, that become qualitative for smaller wave vectors as the slope of $\phi^{s}(t)$ versus $F_{\text {ext }}$ describes a maximum (explained in Sec. V A), while the true critical amplitude grows continuously with decreasing $q$.

In the simulations, the prefactor of the $t^{-1 / 2}$ behavior in the tracer position correlation function has been estimated by averaging the value of $t^{1 / 2} \phi_{q}(t)$ in the interval $t \in[10,25] d^{2} / D_{0}$. The results are presented again in comparison with the estimation from the long-time value of $\phi^{s}(t)$ versus $F_{\text {ext }}$ in Fig. 8. As in the previous case, the data have been matched at the largest wave vector in the perpendicular direction. Again, the overall behaviour is similar between both estimates of the critical amplitude for large wave vectors, but differences appear at small ones. The estimation from $t^{1 / 2} \phi_{q}(t)$ shows a modulation with $q$, which is not observed in the theory or in the other estimation of the critical amplitude, but which is almost within the error bars of the data. The difference in magnitude between the critical amplitudes obtained from theory and simulations can be related to the observation that the transition between localized and delocalized behavior occurs in a much more narrow force range in the theory than in the simulations. Hence, the slopes are steeper and the critical amplitude is larger.

In summary, we find a critical power-law decay with exponent $-1 / 2$ for the tracer position correlation function at long times in theory and simulations. This behavior is screened at small wave vectors. The force associated with the critical behavior is consistent with the critical force obtained in the previous section.

\section{Average tracer displacement}

Both, the analysis of the simulations and theory at a finite time show that the region of small wave vectors is problematic 


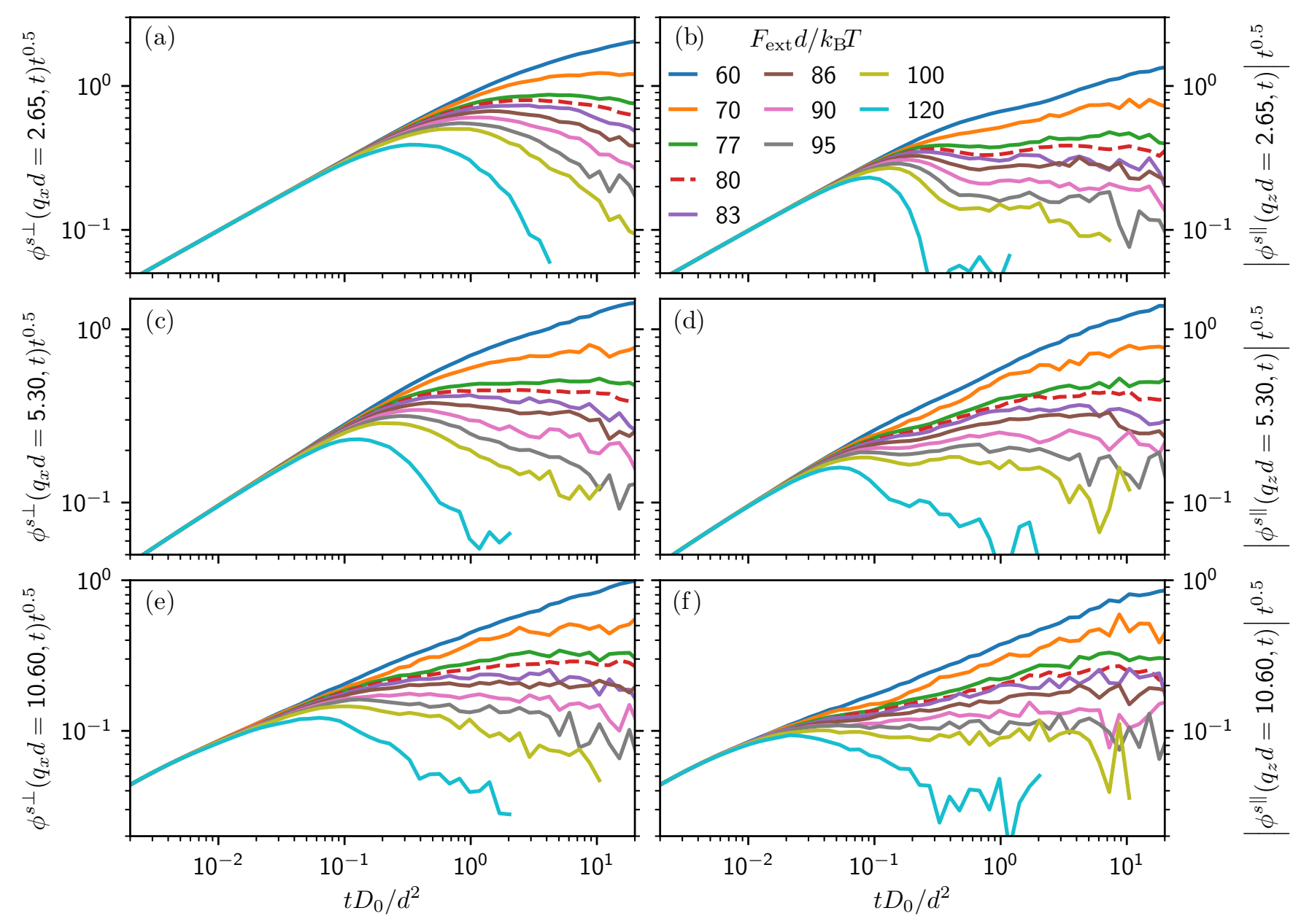

FIG. 10. Rectification plots for the tracer position correlation function for wave vectors perpendicular (left panels) and parallel to the external force (right panels) from simulations. Several forces around the critical one are presented; from top to bottom: $F_{\text {ext }}=60,70,77,80$ (dashed line), 83, 86, 90, 95, 100, and $120 k_{\mathrm{B}} T / d$. Different wave vectors are presented: $q d=2.65$ (top panels), $q d=5.30$ (intermediate panels) and $q d=10.60$ (bottom panels).

because the tracer position correlation function decays very slowly. In the simulations, this is an important problem, as the simulation time is always finite, restricting the wave vectors that can be analyzed. In the theory, on the other hand, this regime has to be handled with care as well to ensure that both (i) the long time limit (either zero in the delocalized state or finite in the localized one) and (ii) the small-wave-vector behavior are correctly treated in the numerical integration scheme, because all modes are coupled. Since the average tracer displacement and mean-squared displacement (MSD) perpendicular and parallel to the external force, are obtained from the small-wave-vector limit of the correlation function, this problem prevents us from analyzing the results of the model for these quantities.

The present model predicts, based on the results for the schematic model, that the tracer displacement grows according to a power law with exponent $1 / 2,\langle\delta z\rangle \sim t^{1 / 2}$, for the critical force, separating the localized regime (where $\langle\delta z\rangle$ approaches a constant at long times) from the delocalized regime (where $\langle\delta z\rangle$ grows linearly). For the MSD in the direction perpendicular to the force, a similar power law is found, while in the parallel direction, it is predicted that $\left\langle\Delta z^{2}\right\rangle$ grows linearly, for the critical force.
These predictions for the tracer displacements cannot be confirmed in the simulations, as shown by the rectification plots in Fig. 11. The average tracer displacement grows faster than the predicted critical behavior for all forces, and in particular, at the critical force, it grows approximately linearly (for smaller forces it grows sublinear). Similarly, the mean squared displacement both in the parallel and perpendicular directions do not follow the predicted behaviour for the critical force (intermediate and bottom panels of Fig. 11). The MSD in the perpendicular plane grows approximately linearly with time around and above the critical force, while in the force direction, superdiffusion is observed (i.e., $\left\langle\Delta z^{2}\right\rangle \sim t^{-2 \alpha}$ with $-2 \alpha>1$ ) for forces above the critical one. A close look at the local exponents (center column in Fig. 11) hints a plateau at long times, from where we can read off an exponent of approximately 0.9 for the mean displacement and the variance perpendicular to the force and an exponent of 1.8 for the variance in the force direction. These absolute values do not agree with the predictions from theory. Still, their ratios follow the prediction that the exponent of the power law of the variance in the force direction should be twice as large as for the two other quantities. The difference between theory and simulations is not understood. It could possibly arise 

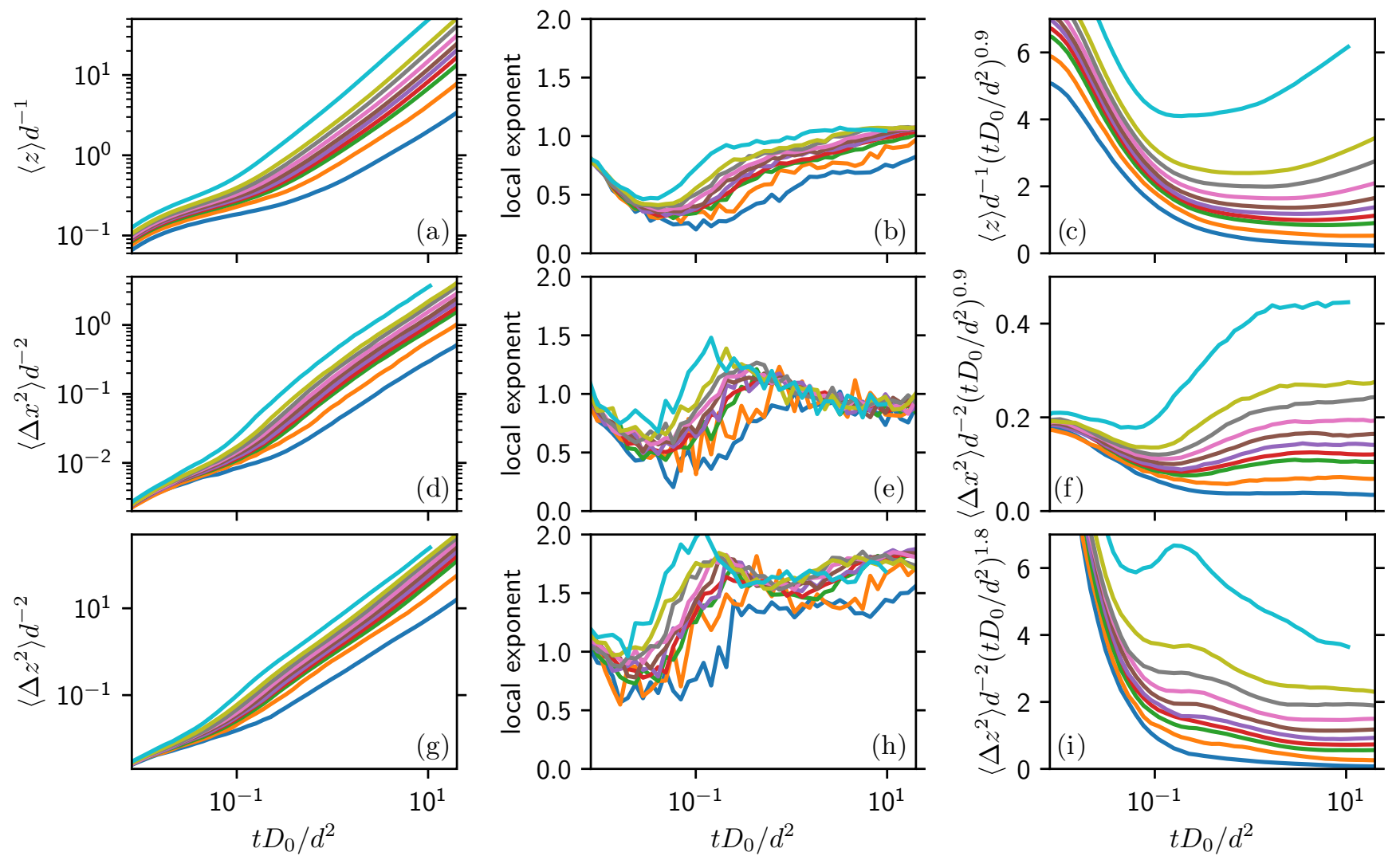

FIG. 11. Critical laws for mean displacement (top row), variance $\left\langle\Delta x^{2}\right\rangle$ perpendicular to the force (center row), and variance $\left\langle\Delta z^{2}\right\rangle$ parallel to the force (bottom row) in the simulations for different forces (color code as in Fig. 10). The left column shows the evolution, the center row their local exponent and the right row the rectified moments scaled with exponent 0.9 for the mean displacement and the perpendicular variance and 1.8 for the variance parallel to the force.

from the instability in the numerical solutions arising from the small wave-vector divergence of the critical amplitude (changing the $q$-discretization changes $\alpha$ [25]), but also from the avoided glass transition in the simulations, which modifies the behavior of the mean tracer trajectory, as shown previously [22].

To summarize this section, it is difficult to pinpoint the critical force based on the critical scaling laws for the moments. First, there is little variation in the local exponent for different forces. Second, the exponents do not coincide with the theoretical prediction, but are slightly larger. Nevertheless, the relation between the exponents for the different moments matches the theoretical prediction.

\section{Stationary velocity}

In previous works, the average velocity was analyzed [18], given by $\bar{v}=\Delta z / \Delta t$, where $\Delta z$ is the displacement of the probe at the end of the experiment and $\Delta t$ its duration. For long times, this converges to the definition of the stationary velocity $v_{\text {st }}$ as introduced in the paragraph before Eq. (25). To assess the effects of finite waiting times we calculate $\langle z\rangle(t) / t$ in Fig. 12 for different values of $t$ from our theory. We observe that the final velocity is overestimated below the critical force and underestimated above the critical force. This smears out the predicted linear behavior $v_{\text {st }} \propto \delta$ for $\delta>0$. For $t D_{0} / d^{2}>10^{2}$ this linear relation is nicely visible as indicated by the dashed/dotted line. This yields another criterion to identify a lower limit for the critical force. As the velocity is underestimated for forces above the critical force, we will

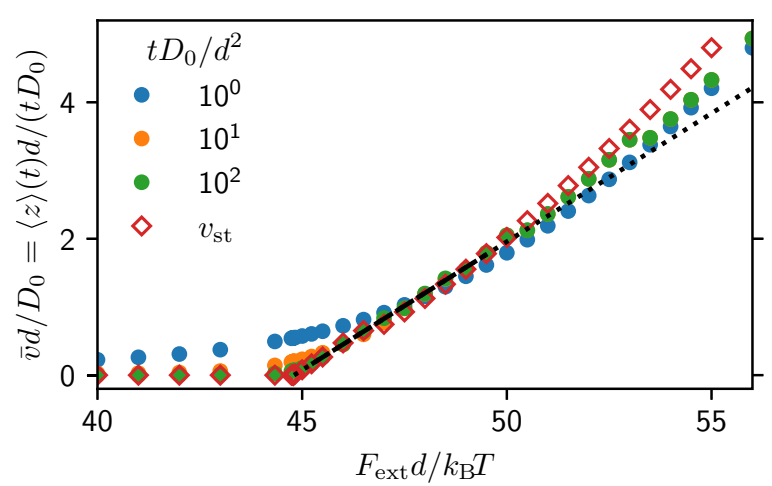

FIG. 12. Average velocity for the theoretical calculations for different waiting times $t$ (circles). The stationary velocity $v_{\mathrm{st}}$ (open diamond) is calculated from the memory relation Eq. (25). The dashed line is a linear fit to the data for $t d^{2} / D_{0}=10^{2}$ in the range $45<F_{\text {ext }} d / k_{\mathrm{B}} T<50$, which is extrapolated for the full range (dotted). This result was obtained using the grid with 25 points in the nonuniform part. 


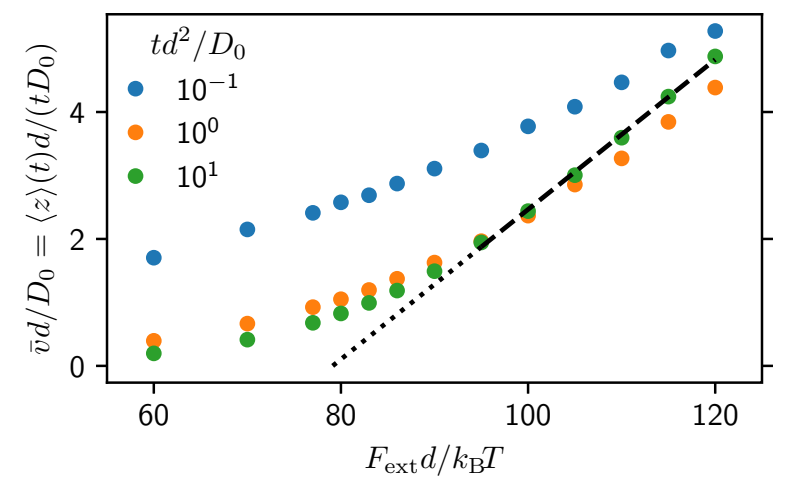

FIG. 13. Average velocity for the simulations for different waiting times $t$ (circles). The dashed line is a linear fit to the data for $t D_{0} / d^{2}=10^{1}$ and $85<F_{\text {ext }} d / k_{\mathrm{B}} T$, which is extrapolated for the full range (dotted).

always obtain a value for the $x$ intercept which is smaller than the true critical force.

Furthermore, we also test the prediction for the stationary velocity based on the memory integral Eq. (25). These results are shown as open diamonds in Fig. 12. They exhibit the same predicted behavior close to the critical force, but deviate for larger forces. The differences can be attributed to numerical artifacts in connection with the stability of the solution algorithm (see Chap. 3 of Ref. [25] for details).

The average velocity from the simulations is analyzed in Fig. 13. Again, we test for finite time effects (circles) and find a good agreement for $t=10^{0}$ and $t=10^{1}$. The average velocities cross at a force of about $90 k_{\mathrm{B}} T / d$ similar to the results of the theory. Above this crossing, we can identify a linear behavior. A linear fit of these data gives a lower bound for the critical force of about $F_{c}=(79 \pm 3) k_{\mathrm{B}} T / d$, which is again compatible with the previous results. As before, the range in which the velocity increases is much broader than in the theoretical calculations and the transition is less sharp than in theory.

Concluding this section, we find that the critical force can be estimated by extrapolating the average velocity linearly to zero, when the duration of the experiment is long enough.

\section{CONCLUSIONS}

Active microrheology provides access to the microscopic properties of a complex fluid by the action of a microscopic stress, typically exerted onto a single colloidal bead. When the host system is beyond the glass transition, a finite stress must be applied to make the system flow, or in microrheology, to delocalize the tracer. The properties of this delocalization transition are studied here for a glass of colloidal hard spheres with theory and simulations. The theory model is based on the Smoluchowski equation, where the glass is described with mode-coupling theory, and a schematic model is also provided which contains only three correlators, dropping the dependence on the wave vector but retaining the direction dependence, and with a simplified memory kernel. The model predicts that the tracer is indeed localized for small forces by the cage of its neighbours, but it can break free above a threshold force, which depends on the couplings with the bath and external force. This delocalization transition has the properties of an A-type transition within mode-coupling theory, making it difficult to observe in simulations and experiments, but also tricky in the numerical solution of the theory equations. However, some properties of the transition offer consistency checks, that have here been tested with Langevin dynamics simulations.

The theory predictions indicate that, in the localized regime but close to the delocalization transition, the nonergodicity parameter decays linearly with the pulling force, reaching the zero-axis at the critical force. There, the tracer position correlation function decays with the square root of time, with an amplitude that is proportional to the slope of the nonergodicity parameter versus force. While these results are based on the assumption that the time is large enough, we confirm that this predictions can also be tested when the values at a finite time are taken. Concomitant power laws follow for the average displacement and mean squared displacement, which are observed in the simulations, albeit with different exponents.

As a result, we confirm three methods to determine the critical force for the delocalization transition. The first method is based on linearly extrapolating the values of the tracer position correlation functions at long times as a function of force. The second method uses the appearance of a critical power law with exponent $1 / 2$ close to the critical force. The last method is based on a linear extrapolation of the average velocity of the probe. The first two methods yield the same critical force if the wave vectors used are not too small. For small wave vectors we find some anomalies, like the divergence of the critical amplitude and a shadowing of the critical power law. The last method requires sufficiently long experimental times. While the predicted behavior for all three methods is applicable only in a very narrow force window in the theoretical calculations, we find these signatures in the simulations over a much broader window of forces, which makes them even more suitable for applications in experimental systems.

The critical dynamics we derived and tested holds universally at a depinning transition from local cages as described by mode-coupling theory. At the considered delocalization transition, the vanishing of the arrested component $f_{q}^{s}$ is accompanied by the divergence of the critical amplitude for small wave vectors; see Fig. 6. Considering the orientational motion of an anisotropic particle pinned in a glass host, a finite critical amplitude can be anticipated [35]. Thus, testing orientational microrheology, where an external torque is applied to an elongated probe particle in a glassy host would ideally be suited to test the predicted critical dynamics.

\section{ACKNOWLEDGMENTS}

We thank Gustavo Abade for his initial implementation of the algorithm to solve the dynamics of the theory. M.G. and M.F. acknowledge support from project B5 of SFB1214. A.M.P. acknowledges financial support from the Spanish Ministerio de Ciencia under Project no. PGC2018-101555-B-I00. 


\section{APPENDIX A: ABBREVIATIONS FOR THE SCHEMATIC} MODEL

In this section, we give the abbreviations for the betascaling analysis of the schematic model:

$$
\begin{aligned}
V_{\|}^{\mathrm{re}} & =\frac{f_{b}}{F_{\mathrm{ext}}^{2} \kappa_{\|}^{2}+1}\left(\begin{array}{c}
v_{1}^{s} \\
F_{\mathrm{ext}} \kappa_{\|} v_{1}^{s} \\
v_{2}^{s}
\end{array}\right), \\
V_{\|}^{\mathrm{im}} & =\frac{f_{b}}{F_{\mathrm{ext}}^{2} \kappa_{\|}^{2}+1}\left(\begin{array}{c}
F_{\mathrm{ext}} \kappa_{\|} v_{1}^{s} \\
-v_{1}^{s} \\
F_{\mathrm{ext}} \kappa_{\|} v_{2}^{s}
\end{array}\right), \\
W_{\perp} & =f_{b}^{2}\left(\begin{array}{ccc}
\left(v_{2}^{s}\right)^{2} & 0 & \frac{v_{1}^{s} v_{2}^{s}}{2} \\
0 & \left(v_{2}^{s}\right)^{2} & 0 \\
\frac{v_{1}^{s} v_{2}^{s}}{2} & 0 & 0
\end{array}\right), \\
V_{\perp} & =\tau_{3} f_{b}\left(\begin{array}{cc}
v_{2}^{s} & \\
-\kappa_{\perp} F_{\mathrm{ext}} v_{2}^{s} \\
v_{1}^{s}
\end{array}\right), \\
U_{\perp} & =v_{2}^{s} f_{b}\left(\begin{array}{c}
1 \\
\kappa_{\perp} F_{\mathrm{ext}} \\
0
\end{array}\right),
\end{aligned}
$$

\section{APPENDIX B: CRITICAL FORCE OF THE SCHEMATIC MODEL}

In this section, we will show how to derive an analytical expression for the critical force and the critical amplitude of the schematic model defined in Eqs. (7)-(10) for the case $\kappa_{\|}=\kappa_{\perp}=: \kappa$. The starting point is the iteration equations for the critical amplitude Eq. (18). For the sake of simplicity, we replace $v_{1}^{s}$ by $\beta v_{2}^{s}$. The iteration equations then read

$$
\begin{aligned}
& h_{1}=\frac{f v_{2}^{s}}{F_{\mathrm{ext}}^{2} \kappa^{2}+1}\left(F_{\mathrm{ext}} \kappa \beta h_{2}+\beta h_{1}+h_{3}\right), \\
& h_{2}=\frac{f v_{2}^{s}}{F_{\mathrm{ext}}^{2} \kappa^{2}+1}\left(F_{\mathrm{ext}} \kappa\left(\beta h_{1}+h_{3}\right)-\beta h_{2}\right), \\
& h_{3}=f v_{2}^{s} \frac{\beta h_{1} h_{3}+h_{1}^{2}+h_{2}^{2}}{F_{\mathrm{ext}} \kappa h_{2}+h_{1}} .
\end{aligned}
$$

First, we want to rewrite the denominator in Eq. (B1c). By combining Eqs. (B1a) and (B1b), we find

$$
h_{1}+F_{\text {ext }} \kappa h_{2}=f v_{2}^{s}\left(\beta h_{1}+h_{3}\right) .
$$

Substituting this back into Eq. (B1c), we get

$$
h_{3}=\frac{\beta h_{1} h_{3}+h_{1}^{2}+h_{2}^{2}}{\beta h_{1}+h_{3}} \Leftrightarrow h_{3}^{2}=h_{1}^{2}+h_{2}^{2} .
$$

Using again Eqs. (B1a) and (B1b) we can find an expression for the right-hand side:

$$
h_{1}^{2}+h_{2}^{2}=\frac{\left(f v_{2}^{s}\right)^{2}}{F_{\mathrm{ext}}^{2} \kappa^{2}+1}\left(\beta^{2}\left(h_{1}^{2}+h_{2}^{2}\right)+2 \beta h_{1} h_{3}+h_{3}^{2}\right) .
$$

Solving this equation for $h_{1}^{2}+h_{2}^{2}$, we get

$$
h_{1}^{2}+h_{2}^{2}=\frac{\left(f v_{2}^{s}\right)^{2}\left(2 \beta h_{1} h_{3}+h_{3}^{2}\right)}{F_{\mathrm{ext}}^{2} \kappa^{2}+1-\left(f v_{2}^{s}\right)^{2} \beta^{2}} .
$$

Now this equation is substituted into Eq. (B3) leading to

$$
h_{3}^{2}=\frac{\left(f v_{2}^{s}\right)^{2}\left(2 \beta h_{1} h_{3}+h_{3}^{2}\right)}{F_{\mathrm{ext}}^{2} \kappa^{2}+1-\left(f v_{2}^{s}\right)^{2} \beta^{2}} .
$$

Here, we have eliminated the variable $h_{2}$. In the next step we make use of the fact, that the iteration equation is scale invariant, i.e., any multiple of a solution is again a solution. This allows us to fix $h_{3}=1$ and we obtain a linear equation for $h_{1}$. Its solution is

$$
h_{1}=\frac{F_{\mathrm{ext}}^{2} \kappa^{2}+1}{2 \beta\left(f v_{2}^{s}\right)^{2}}-\frac{1+\beta^{2}}{2 \beta} .
$$

Inserting this solution together with $h_{3}=1$ into Eqs. (B1a) and (B1b), we get two equations to be solved for $h_{2}$

$$
\begin{aligned}
h_{2}= & \left\{F_{\mathrm{ext}}^{4} \kappa^{4}+\left(f v_{2}^{s}\right)^{3} \beta\left(\beta^{2}-1\right)-1\right. \\
& \left.-\left(F_{\mathrm{ext}}^{2} \kappa^{2}+1\right)\left[\left(f v_{2}^{s}\right)^{2}\left(\beta^{2}+1\right)+\beta f v_{2}^{s}-2\right]\right\} \\
& \times\left[2 F_{\mathrm{ext}} \kappa \beta^{2}\left(f v_{2}^{s}\right)^{3}\right]^{-1}, \\
h_{2}= & \frac{F_{\mathrm{ext}} \kappa}{2 f v_{2}^{s}} \frac{F_{\mathrm{ext}}^{2} \kappa^{2}+1+\left(f v_{2}^{s}\right)^{2}\left(1-\beta^{2}\right)}{F_{\mathrm{ext}}^{2} \kappa^{2}+\beta f v_{2}^{s}+1} .
\end{aligned}
$$

Since both equations must give the same result at the critical force, we can obtain an equation for the critical force by equating these two equations:

$$
\begin{aligned}
0= & F_{\mathrm{ext}}^{6} \kappa^{6}+F_{\mathrm{ext}}^{4} \kappa^{4}\left[3-\left(f v_{2}^{s}\right)^{2}\left(1+2 \beta^{2}\right)\right] \\
& +F_{\mathrm{ext}}^{2} \kappa^{2}\left[\left(f v_{2}^{s}\right)^{4}\left(\beta^{4}-\beta^{2}\right)-2 \beta\left(f v_{2}^{s}\right)^{3}\right. \\
& \left.-\left(f v_{2}^{s}\right)^{2}\left(4 \beta^{2}+2\right)+3\right]+\left(f v_{2}^{s}\right)^{4}\left(\beta^{4}-\beta^{2}\right) \\
& -2 \beta\left(f v_{2}^{s}\right)^{3}-\left(f v_{2}^{s}\right)^{2}\left(2 \beta^{2}+1\right)+1 .
\end{aligned}
$$

This is a third order polynomial in $F_{\text {ext }}^{2} \kappa^{2}$, which has the following three solutions:

$$
\left\{-1,\left(f v_{2}^{s}\right)^{2}\left(\beta^{2}+\frac{1}{2}\right)-1 \pm \frac{\left(f v_{2}^{s}\right)^{2}}{2} \sqrt{8 \beta^{2}+1+\frac{8 \beta}{f v_{2}^{s}}}\right\} .
$$

As we want the critical force to be real, we have to choose the largest (positive) solution, which is

$$
F_{c}=\frac{1}{\kappa}\left[\frac{\left(f v_{2}^{s}\right)^{2}}{2}\left(2 \beta^{2}+1+\sqrt{8 \beta^{2}+1+\frac{8 \beta}{f v_{2}^{s}}}\right)-1\right]^{\frac{1}{2}} .
$$

This solution can now be plugged into Eqs. (B7) and (B8b) to find

$$
\begin{aligned}
& h_{1}=\frac{1}{4 \beta}\left(\sqrt{8 \beta^{2}+1+\frac{8 \beta}{f v_{2}^{s}}}-1\right), \\
& h_{2}=\frac{1}{2} \frac{F_{c} \kappa\left(3+\sqrt{8 \beta^{2}+1+8 \beta / f v_{2}^{s}}\right)}{f v_{2}^{s}\left(2 \beta^{2}+1+\sqrt{8 \beta^{2}+1+8 \beta / f v_{2}^{s}}\right)+2 \beta} .
\end{aligned}
$$


By definition, we had $h_{3}=1$. Finally, one has to replace $\beta$ by $v_{1}^{s} / v_{2}^{s}$ to get the result in terms of $v_{1}^{s}$ and $v_{2}^{s}$.

\section{APPENDIX C: SCALING LAW FOR THE SCHEMATIC MODEL}

In this section, we give some arguments why the timescale for the decay close to the critical point scales like the inverse distance from the critical point. We start with the factorization ansatz $\phi_{i}(s)=h_{i} g(s)$ with the critical amplitudes $h_{i}$ and a general time dependency $g(s)$, which is inserted into the equations of motion close to the critical point given in Eq. (15). One can identify the stability matrix $S^{c}$ [given in Eq. (14)] so that the equations of motion read

$$
\begin{aligned}
g(s) \sum_{i} S^{c}(\boldsymbol{h})_{1 i} h_{i}= & g(s) \Delta V_{\|}^{\mathrm{re}}(\boldsymbol{h})+\tau_{1} \\
& -s[g(s)]^{2}\left(h_{1}^{2}-h_{2}^{2}\right) \\
g(s) \sum_{i} S^{c}(\boldsymbol{h})_{2 i} h_{i}= & g(s) \Delta V_{\|}^{\mathrm{im}}(\boldsymbol{h})+\tau_{2} \\
& -s[g(s)]^{2} 2 h_{1} h_{2} \\
g(s) \sum_{i} S^{c}(\boldsymbol{h})_{3 i} h_{i}= & 2\left\{g(s)\left[\Delta \frac{\mathcal{W}}{\mathcal{U}}(\boldsymbol{h})\right]+\frac{\mathcal{V}(\boldsymbol{h})}{\mathcal{U}(\boldsymbol{h})}\right. \\
& \left.-\tau_{3} \frac{\mathcal{W}(\boldsymbol{h})}{[\mathcal{U}(\boldsymbol{h})]^{2}}+\tau_{3}-s[g(s)]^{2} h_{3}^{2}\right\},
\end{aligned}
$$

with the definitions

$$
\begin{aligned}
\Delta V_{\|}^{\mathrm{re} / \mathrm{im}}(\boldsymbol{h}) & :=\sum_{i=1}^{3}\left[\left(V_{\|}^{\mathrm{re} / \mathrm{im}}\right)_{i}-\left(V_{\|}^{\mathrm{re} / \mathrm{im}, c}\right)_{i}\right] h_{i}, \\
\mathcal{W}(\boldsymbol{h}) & :=\sum_{i, j=1}^{3}\left(W_{\perp}\right)_{i j} h_{i} h_{j} \\
\mathcal{U}(\boldsymbol{h}) & :=\sum_{i=1}^{3}\left(U_{\perp}\right)_{i} h_{i} \\
\mathcal{V}(\boldsymbol{h}) & :=\sum_{i=1}^{3}\left(V_{\perp}\right)_{i} h_{i} \\
\Delta \frac{\mathcal{W}}{\mathcal{U}}(\boldsymbol{h}) & :=\frac{\mathcal{W}^{c}(\boldsymbol{h})}{\mathcal{U}^{c}(\boldsymbol{h})}-\frac{\mathcal{W}(\boldsymbol{h})}{\mathcal{U}(\boldsymbol{h})}
\end{aligned}
$$

where the superscript $c$ labels the vertices at the critical force. As the critical stability matrix $S^{c}$ is by definition not invertible at the critical point, this system of equations only has a solution if the right-hand side lies in the invertible subspace (the image) of $S^{c}$. This can be achieved by adjusting the amplitude of $\boldsymbol{h}$ such that the projection of the right-hand side onto the left zero-eigenvector $\tilde{\boldsymbol{h}}$ of $S^{c}$ vanishes. $\tilde{\boldsymbol{h}}$ is given by the determining equation $\tilde{\boldsymbol{h}}^{T} S^{c}=0$ or equivalently $\left(S^{c}\right)^{T} \tilde{\boldsymbol{h}}=$ 0 . Hence, the solution condition reads

$$
0=\tilde{c}_{1}+g(s) \alpha \tilde{c}_{2}+s(g(s))^{2} \alpha^{2} \tilde{c}_{3},
$$

with the abbreviations

$$
\begin{aligned}
& \tilde{c}_{1}=\tilde{h_{1}} \tau_{1}+\tilde{h_{2}} \tau_{2}+2 \tilde{h_{3}}\left\{\frac{\mathcal{V}(\boldsymbol{h})}{\mathcal{U}(\boldsymbol{h})}-\tau_{3} \frac{\mathcal{W}(\boldsymbol{h})}{[\mathcal{U}(\boldsymbol{h})]^{2}}+\tau_{3}\right\}, \\
& \tilde{c}_{2}=\tilde{h_{1}} \Delta V_{\|}^{\mathrm{re}}(\boldsymbol{h})+\tilde{h_{2}} \Delta V_{\|}^{\mathrm{im}}(\boldsymbol{h})+2 \tilde{h}_{3} \Delta \frac{\mathcal{W}}{\mathcal{U}}(\boldsymbol{h}), \\
& \tilde{c}_{3}=\tilde{h_{1}}\left(h_{1}^{2}-h_{2}^{2}\right)+\tilde{h_{2}} h_{1} h_{2}-2 \tilde{h}_{3} h_{3}^{2} .
\end{aligned}
$$

This is a quadratic equation for $\alpha$, which can be solved explicitly. For the following, we assume that $\boldsymbol{h}$ is normalized such that $\alpha=1$. Note that $\tilde{c}_{3}$ is independent of the external force and $\tilde{c}_{2}$ is proportional to the distance from the critical force to lowest order. Then, it remains to find a solution of the following equation (expanded to lowest order in $\delta$, the distance to the critical force)

$$
0=c_{1}+\delta c_{2} g(s)+s[g(s)]^{2},
$$

with $c_{1} \tilde{c}_{3}=\tilde{c}_{1}^{c}$ and $c_{2} \tilde{c}_{3}=\left.\frac{\partial}{\partial F_{\text {ext }}} \tilde{c}_{2}\right|_{F_{c}}$.

This equation leads to the following scaling argument: Inserting the ansatz $g(s):=\sigma g_{ \pm}(\tau s)$ into Eq. (C5) gives a scale-independent equation,

$$
0=c_{1} \pm c_{2} \delta \sigma g_{ \pm}(\tau s)+\frac{\sigma^{2}}{\tau} \tau s\left[g_{ \pm}(\tau s)\right]^{2},
$$

if and only if $\sigma|\delta|=1$ and $\sigma^{2} / \tau=1 . g_{ \pm}(t)$ are then the solutions of this scale-independent equation. Hence, we conclude

$$
g(s)=|\delta|^{-1} g_{ \pm}\left(s \delta^{-2}\right) \quad \text { for } \delta \gtrless 0 .
$$

This is the same scaling equation as in conventional schematic MCT models (see Refs. [24,35,36]).

\section{APPENDIX D: SCALING OF THE VELOCITY MEMORY KERNEL}

The schematic model exhibits a timescale $\tau=\delta^{-2}$ for the behavior close to the critical force. The same scaling can be observed in the numerical solution of the full model as shown in Fig. 9. This justifies the assumption of a factorization of the correlators, i.e., $\boldsymbol{\phi}^{s}(t)=\boldsymbol{h} g(s)$ with the same scaling function as for the schematic model, i.e., $g(s)=\delta^{-1} g_{+}\left(s \delta^{-2}\right)$ [above the critical force, see Eq. (C7)], we find

$$
\begin{aligned}
\int_{0}^{\infty} m_{0}^{z z}\left(t^{\prime}\right) d t^{\prime} & =\lim _{s \rightarrow 0} m_{0}^{z z}(s)=\lim _{s \rightarrow 0} m_{0}^{z z}[\boldsymbol{h} g(s)] \\
& =m_{0}^{z z}[\boldsymbol{h}] \lim _{s \rightarrow 0} \delta^{-1} g_{+}\left(s \delta^{-2}\right) \\
& =m_{0}^{z z}[\boldsymbol{h}] \delta^{-1} g_{+}(0),
\end{aligned}
$$

where we used the linearity of the memory kernel functional $m_{0}^{z z}$ to separate the time dependency. This yields $\int_{0}^{\infty} m_{0}^{z z}\left(t^{\prime}\right) d t^{\prime} \propto|\delta|^{-1}$ close to the critical force.

\section{APPENDIX E: STABILITY MATRIX OF THE FULL MODEL}

Separating the root problem Eq. (23) into real and imaginary parts, we obtain

$$
\begin{aligned}
& J_{q}^{\prime \mathrm{re}}\left(\boldsymbol{v}^{\mathrm{re}}, \boldsymbol{v}^{\mathrm{im}}\right)=v_{q}^{\mathrm{re}}-\frac{a^{\mathrm{re}}\left(a^{\mathrm{re}}+b^{\mathrm{re}}\right)+a^{\mathrm{im}}\left(a^{\mathrm{im}}+b^{\mathrm{im}}\right)}{\left(a^{\mathrm{re}}+b^{\mathrm{re}}\right)^{2}+\left(a^{\mathrm{im}}+b^{\mathrm{im}}\right)^{2}}, \\
& J_{q}^{\mathrm{im}}\left(\boldsymbol{v}^{\mathrm{re}}, \boldsymbol{v}^{\mathrm{im}}\right)=v_{q}^{\mathrm{im}}-\frac{a^{\mathrm{im}} b^{\mathrm{re}}-a^{\mathrm{re}} b^{\mathrm{im}}}{\left(a^{\mathrm{re}}+b^{\mathrm{re}}\right)^{2}+\left(a^{\mathrm{im}}+b^{\mathrm{im}}\right)^{2}},
\end{aligned}
$$


with $v_{q}^{\text {re }}:=\operatorname{Re} v_{q}, v_{q}^{\text {im }}=\operatorname{Im} v_{q}$ and

$$
\begin{aligned}
& a^{\mathrm{re}}=\operatorname{Re} \boldsymbol{v}^{T} A_{q} \boldsymbol{v}, \\
& b^{\mathrm{re}}=\operatorname{Re} B_{q}^{T} \boldsymbol{v},
\end{aligned}
$$

$$
\begin{aligned}
& a^{\mathrm{im}}=\operatorname{Im} \boldsymbol{v}^{T} A_{q} \boldsymbol{v}, \\
& b^{\mathrm{im}}=\operatorname{Im} B_{q}^{T} \boldsymbol{v} .
\end{aligned}
$$

The reduced stability matrix (i.e., the Jacobian of the nontrivial part) then consists of the four blocks

$$
\begin{aligned}
& \frac{\partial J_{q}^{\prime \mathrm{re}}}{\partial v_{p}^{\mathrm{re} / \mathrm{im}}}=c\left(\left(\frac{\partial a^{\mathrm{re}}}{\partial v_{p}^{\mathrm{re} / \mathrm{im}}}\right)\left[2 a^{\mathrm{im}} b^{\mathrm{im}} a^{\mathrm{re}}-\left(a^{\mathrm{im}}\right)^{2} b^{\mathrm{re}}+b^{\mathrm{re}}\left(a^{\mathrm{re}}+b^{\mathrm{re}}\right)^{2}+\left(b^{\mathrm{im}}\right)^{2}\left(2 a^{\mathrm{re}}+b^{\mathrm{re}}\right)\right]\right. \\
& +\left(\frac{\partial a^{\mathrm{im}}}{\partial v_{p}^{\mathrm{re} / \mathrm{im}}}\right)\left\{b^{\mathrm{im}}\left[\left(a^{\mathrm{im}}\right)^{2}+\left(b^{\mathrm{im}}\right)^{2}-\left(a^{\mathrm{re}}\right)^{2}+\left(b^{\mathrm{re}}\right)^{2}\right]+2 a^{\mathrm{im}}\left[\left(b^{\mathrm{im}}\right)^{2}+b^{\mathrm{re}}\left(a^{\mathrm{re}}+b^{\mathrm{re}}\right)\right]\right\} \\
& +\left(\frac{\partial b^{\mathrm{re}}}{\partial v_{p}^{\mathrm{re} / \mathrm{im}}}\right)\left\{-2 a^{\mathrm{im}} b^{\mathrm{im}} b^{\mathrm{re}}-\left(a^{\mathrm{im}}\right)^{2}\left(a^{\mathrm{re}}+2 b^{\mathrm{re}}\right)-a^{\mathrm{re}}\left[-\left(b^{\mathrm{im}}\right)^{2}+\left(a^{\mathrm{re}}+b^{\mathrm{re}}\right)^{2}\right]\right\} \\
& \left.+\left(\frac{\partial b^{\mathrm{im}}}{\partial v_{p}^{\mathrm{re} / \mathrm{im}}}\right)\left\{-\left(a^{\mathrm{im}}\right)^{3}-2\left(a^{\mathrm{im}}\right)^{2} b^{\mathrm{im}}-2 b^{\mathrm{im}} a^{\mathrm{re}}\left(a^{\mathrm{re}}+b^{\mathrm{re}}\right)-a^{\mathrm{im}}\left[\left(b^{\mathrm{im}}\right)^{2}+\left(a^{\mathrm{re}}\right)^{2}-\left(b^{\mathrm{re}}\right)^{2}\right]\right\}\right), \\
& \frac{\partial J_{q}^{\prime \text { im }}}{\partial v_{p}^{\mathrm{re} / \mathrm{im}}}=c\left(\left(\frac{\partial a^{\mathrm{re}}}{\partial v_{p}^{\mathrm{re} / \mathrm{im}}}\right)\left\{b^{\mathrm{im}}\left[\left(a^{\mathrm{re}}\right)^{2}-\left(a^{\mathrm{im}}\right)^{2}-\left(b^{\mathrm{im}}\right)^{2}-\left(b^{\mathrm{re}}\right)^{2}\right]-2 a^{\mathrm{im}}\left[\left(b^{\mathrm{im}}\right)^{2}+b^{\mathrm{re}}\left(a^{\mathrm{re}}+b^{\mathrm{re}}\right)\right]\right\}\right. \\
& +\left(\frac{\partial a^{\mathrm{im}}}{\partial v_{p}^{\mathrm{re} / \mathrm{im}}}\right)\left[2 a^{\mathrm{im}} b^{\mathrm{im}} a^{\mathrm{re}}-\left(a^{\mathrm{im}}\right)^{2} b^{\mathrm{re}}+b^{\mathrm{re}}\left(a^{\mathrm{re}}+b^{\mathrm{re}}\right)^{2}+\left(b^{\mathrm{im}}\right)^{2}\left(2 a^{\mathrm{re}}+b^{\mathrm{re}}\right)\right] \\
& +\left(\frac{\partial b^{\mathrm{re}}}{\partial v_{p}^{\mathrm{re} / \mathrm{im}}}\right)\left\{\left(a^{\mathrm{im}}\right)^{3}+2\left(a^{\mathrm{im}}\right)^{2} b^{\mathrm{im}}+2 b^{\mathrm{im}} a^{\mathrm{re}}\left(a^{\mathrm{re}}+b^{\mathrm{re}}\right)+a^{\mathrm{im}}\left[\left(b^{\mathrm{im}}\right)^{2}+\left(a^{\mathrm{re}}\right)^{2}-\left(b^{\mathrm{re}}\right)^{2}\right]\right\} \\
& +\left(\frac{\partial b^{\mathrm{im}}}{\partial v_{p}^{\mathrm{re} / \mathrm{im}}}\right)\left\{-2 a^{\mathrm{im}} b^{\mathrm{im}} b^{\mathrm{re}}-\left(a^{\mathrm{im}}\right)^{2}\left(a^{\mathrm{re}}+2 b^{\mathrm{re}}\right)-a^{\mathrm{re}}\left[-\left(b^{\mathrm{im}}\right)^{2}+\left(a^{\mathrm{re}}+b^{\mathrm{re}}\right)^{2}\right]\right\},
\end{aligned}
$$

with $c=\left[\left(a^{\mathrm{re}}\right)^{2}+\left(a^{\mathrm{im}}\right)^{2}+\left(b^{\mathrm{re}}\right)^{2}+\left(b^{\mathrm{im}}\right)^{2}\right]^{-2}$. After calculating the derivatives of $a^{\mathrm{re}}, a^{\mathrm{im}}, b^{\mathrm{re}}, b^{\mathrm{im}}$, with respect to the variables $v_{p}^{\mathrm{re}}$ and $v_{p}^{\mathrm{im}}$, we are ready to construct the stability matrix as

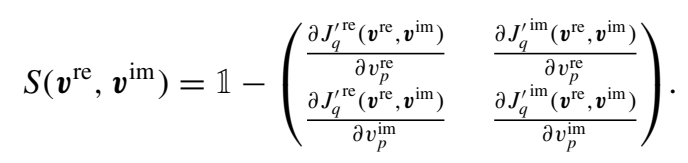

Since $a$ and $b$ are holomorphic functions, there hold the Cauchy-Riemann differential equations as long as $v \neq 0$ :

$$
\frac{\partial J_{q}^{\text {re }}}{\partial v_{p}^{\mathrm{re}}}=\frac{\partial J_{q}^{\prime \mathrm{im}}}{\partial v_{p}^{\mathrm{im}}} \quad \text { and } \quad \frac{\partial J_{q}^{\mathrm{re}}}{\partial v_{p}^{\mathrm{im}}}=-\frac{\partial J_{q}^{\prime \text { im }}}{\partial v_{p}^{\mathrm{re}}} .
$$

The eigenvalues of the reduced stability matrix $\mathbb{1}-S$ can be evaluated numerically and the eigenvalue with the largest modulus is real valued and approaches 1 at the delocalization transition. We also note that this eigenvalue has multiplicity 1 . The value of this largest eigenvalue coincides with the value of the contraction factor determined by the iteration. The eigenvector for this eigenvalue is also called the critical amplitude $\boldsymbol{h}=\left(h_{q}\right)_{q}$ and it determines in classical mode-coupling theory the wave vector dependency of the nonergodicity parameters close to the critical point via

$$
f_{q}-f_{q}^{c}=h_{q} g(\delta)
$$

(p. 239, Eq. (4.78a) in Ref. [24]), where $g(\delta)$ describes the scaling behavior close to the critical force depending on the type of the transition: $g(\delta) \propto \sqrt{|\delta|}$ for a type B transition (p. 245, Eq. (4.91a) in Ref. [24]) and $g(\delta) \propto \delta$ for a type A transition (p. 248, Eq. (4.98) in Ref. [24]).

In the case of vanishing nonergodicity parameters at the critical point, it is also possible to evaluate the eigenvector to the largest eigenvalue of the reduced stability matrix directly from the fixed point iteration scheme. Using (E7) with $f_{q}^{c}=0$ in the iteration equation (21) and dividing by $g(\delta)$ we find in the limit $g(\delta) \rightarrow 0$

$$
h_{q}=\frac{a^{\mathrm{re}}(\boldsymbol{h}) b^{\mathrm{re}}(\boldsymbol{h})-a^{\mathrm{im}}(\boldsymbol{h}) b^{\mathrm{im}}(\boldsymbol{h})+i\left[a^{\mathrm{im}}(\boldsymbol{h}) b^{\mathrm{re}}(\boldsymbol{h})-a^{\mathrm{re}}(\boldsymbol{h}) b^{\mathrm{im}}(\boldsymbol{h})\right]}{\left[b^{\mathrm{re}}(\boldsymbol{h})\right]^{2}+\left[b^{\mathrm{im}}(\boldsymbol{h})\right]^{2}} .
$$


[1] D. Bonn, M. M. Denn, L. Berthier, T. Divoux, and S. Manneville, Yield stress materials in soft condensed matter, Rev. Mod. Phys. 89, 035005 (2017).

[2] P. Sollich, Rheological constitutive equation for a model of soft glassy materials, Phys. Rev. E 58, 738 (1998).

[3] M. L. Falk and J. S. Langer, Dynamics of viscoplastic deformation in amorphous solids, Phys. Rev. E 57, 7192 (1998).

[4] M. Fuchs and M. E. Cates, Theory of Nonlinear Rheology and Yielding of Dense Colloidal Suspensions, Phys. Rev. Lett. 89, 248304 (2002).

[5] T. G. Mason and D. A. Weitz, Linear Viscoelasticity of Colloidal Hard Sphere Suspensions Near the Glass Transition, Phys. Rev. Lett. 75, 2770 (1995).

[6] A. M. Puertas and T. Voigtmann, Microrheology of colloidal systems, J. Phys.: Condens. Matter 26, 243101 (2014).

[7] E. M. Furst and T. M. Squires, Microrheology (Oxford University Press, Oxford, 2017).

[8] A. J. Levine and T. C. Lubensky, One- and Two-Particle Microrheology, Phys. Rev. Lett. 85, 1774 (2000).

[9] T. M. Squires and J. F. Brady, A simple paradigm for active and nonlinear microrheology, Phys. Fluids 17, 073101 (2005).

[10] S. Leitmann, S. Mandal, M. Fuchs, A. M. Puertas, and T. Franosch, Time-dependent active microrheology in dilute colloidal suspensions, Phys. Rev. Fluids 3, 103301 (2018).

[11] I. Gazuz, A. M. Puertas, T. Voigtmann, and M. Fuchs, Active and Nonlinear Microrheology in Dense Colloidal Suspensions, Phys. Rev. Lett. 102, 248302 (2009).

[12] C. F. E. Schroer and A. Heuer, Anomalous Diffusion of Driven Particles in Supercooled Liquids, Phys. Rev. Lett. 110, 067801 (2013).

[13] G. Gradenigo, E. Bertin, and G. Biroli, Field-induced superdiffusion and dynamical heterogeneity, Phys. Rev. E 93, 060105(R) (2016).

[14] R. L. Jack, D. Kelsey, J. P. Garrahan, and D. Chandler, Negative differential mobility of weakly driven particles in models of glass formers, Phys. Rev. E 78, 011506 (2008).

[15] S. R. Williams and D. J. Evans, Linear Response Domain in Glassy Systems, Phys. Rev. Lett. 96, 015701 (2006).

[16] D. Winter, J. Horbach, P. Virnau, and K. Binder, Active Nonlinear Microrheology in a Glass-Forming Yukawa Fluid, Phys. Rev. Lett. 108, 028303 (2012).

[17] C. Reichhardt and C. J. Olson Reichhardt, Active microrheology in active matter systems: Mobility, intermittency, and avalanches, Phys. Rev. E 91, 032313 (2015).

[18] P. Habdas, D. Schaar, A. C. Levitt, and E. R. Weeks, Forced motion of a probe particle near the colloidal glass transition, Europhys. Lett. 67, 477 (2004).

[19] N. Şenbil, M. Gruber, C. Zhang, M. Fuchs, and F. Scheffold, Observation of Strongly Heterogeneous Dynamics at the Depinning Transition in a Colloidal Glass, Phys. Rev. Lett. 122, 108002 (2019).

[20] C. Reichhardt and C. J. O. Reichhardt, Depinning and nonequilibrium dynamic phases of particle assemblies driven over random and ordered substrates: A review, Rep. Prog. Phys. 80, 026501 (2016).

[21] I. Gazuz and M. Fuchs, Nonlinear microrheology of dense colloidal suspensions: A mode-coupling theory, Phys. Rev. E 87, 032304 (2013).

[22] M. Gruber, G. C. Abade, A. M. Puertas, and M. Fuchs, Active microrheology in a colloidal glass, Phys. Rev. E 94, 042602 (2016).

[23] C. J. Harrer, A. M. Puertas, and T. Voigtmann, Probability densities of a forced probe particle on glass: Results from mode-coupling theory and simulations of active microrheology, Z. Phys. Chem. 226, 779 (2012).

[24] W. Götze, Complex Dynamics of Glass-forming Liquids: A Mode-coupling Theory (Oxford University Press, Oxford, 2009).

[25] M. Gruber, Theory of microrheology in complex fluids, Ph.D. thesis, Universität Konstanz, 2019, http://nbn-resolving.de/urn: nbn:de:bsz:352-2-hurftyctohii2.

[26] For a cutoff $q_{\max } d \geqslant 20$ the transition type changes, see Chap. 5 in Ref. [25] .

[27] M. V. Gnann, I. Gazuz, A. M. Puertas, M. Fuchs, and T. Voigtmann, Schematic models for active nonlinear microrheology, Soft Matter 7, 1390 (2011).

[28] We choose as parameters for the $F_{12}$-model $v_{2}=2$ and $v_{1}=2(\sqrt{2}-1)+\varepsilon(\sqrt{2}-1)^{-1}$. This choice of the parameters yields for $\varepsilon>0$ the bath nonergodicity parameter $f_{b}(\varepsilon)=4-$ $2 \sqrt{2}-\varepsilon /(\sqrt{2}-1)+\sqrt{\varepsilon^{2}(3+2 \sqrt{2})+4 \varepsilon(2+\sqrt{2})} / 4$, where $\varepsilon$ determines the distance from the glass transition [21] .

[29] J. K. G. Dhont, An Introduction to Dynamics of Colloids, Studies in Interface Science (Elsevier Science, Amsterdam, 1996).

[30] T. Voigtmann, A. M. Puertas, and M. Fuchs, Tagged-particle dynamics in a hard-sphere system: Mode-coupling theory analysis, Phys. Rev. E 70, 061506 (2004).

[31] F. Weysser, A. M. Puertas, M. Fuchs, and T. Voigtmann, Structural relaxation of polydisperse hard spheres: Comparison of the mode-coupling theory to a Langevin dynamics simulation, Phys. Rev. E 82, 011504 (2010).

[32] A. M. Puertas, Aging of a hard-sphere glass: Effect of the microscopic dynamics, J. Phys.: Condens. Matter 22, 104121 (2010).

[33] W. Paul and D. Y. Yoon, Stochastic phase space dynamics with constraints for molecular systems, Phys. Rev. E 52, 2076 (1995).

[34] W. Götze, Recent tests of the mode-coupling theory for glassy dynamics, J. Phys.: Condens. Matter 11, A1 (1999).

[35] T. Franosch, M. Fuchs, W. Götze, M. R. Mayr, and A. P. Singh, Theory for the reorientational dynamics in glass-forming liquids, Phys. Rev. E 56, 5659 (1997).

[36] S. K. Schnyder, F. Höfling, T. Franosch, and T. Voigtmann, Long-wavelength anomalies in the asymptotic behavior of mode-coupling theory, J. Phys.: Condens. Matter 23, 234121 (2011). 